\title{
Living with ghosts in Hořava-Lifshitz gravity
}

\author{
S. Ramazanov, ${ }^{a}$ F. Arroja, ${ }^{b}$ M. Celoria, ${ }^{a}$ S. Matarrese ${ }^{c, a, d, e}$ and L. Pilo ${ }^{f, g}$ \\ ${ }^{a}$ Gran Sasso Science Institute (INFN), \\ Viale Francesco Crispi 7, I-67100 L'Aquila, Italy \\ ${ }^{b}$ Leung Center for Cosmology and Particle Astrophysics, National Taiwan University, \\ No. 1, Sec. 4, Roosevelt Road, Taipei, 10617 Taipei, Taiwan (R.O.C.) \\ ${ }^{c}$ Dipartimento di Fisica e Astronomia "G. Galilei", Università degli Studi di Padova, \\ via Marzolo 8, I-35131 Padova, Italy \\ ${ }^{d}$ INFN, Sezione di Padova, \\ via Marzolo 8, I-35131 Padova, Italy \\ ${ }^{e}$ INAF-Osservatorio Astronomico di Padova, \\ Vicolo dell'Osservatorio 5, I-35122 Padova, Italy \\ ${ }^{f}$ Dipartimento di Scienze Fisiche e Chimiche, Università di L'Aquila, \\ I-67010 L'Aquila, Italy \\ ${ }^{g}$ INFN, Laboratori Nazionali del Gran Sasso, \\ I-67010 Assergi, Italy \\ E-mail: sabir.ramazanov@gssi.infn.it, arroja@phys.ntu.edu.tw, \\ marco.celoria@gssi.infn.it, sabino.matarrese@pd.infn.it, \\ luigi.pilo@aquila.infn.it
}

AbSTRACT: We consider the branch of the projectable Hořava-Lifshitz model which exhibits ghost instabilities in the low energy limit. It turns out that, due to the Lorentz violating structure of the model and to the presence of a finite strong coupling scale, the vacuum decay rate into photons is tiny in a wide range of phenomenologically acceptable parameters. The strong coupling scale, understood as a cutoff on ghosts' spatial momenta, can be raised up to $\Lambda \sim 10 \mathrm{TeV}$. At lower momenta, the projectable Hořava-Lifshitz gravity is equivalent to General Relativity supplemented by a fluid with a small positive sound speed squared $\left(10^{-42} \lesssim\right) c_{s}^{2} \lesssim 10^{-20}$, that could be a promising candidate for the Dark Matter. Despite these advantages, the unavoidable presence of the strong coupling obscures the implementation of the original Hořava's proposal on quantum gravity. Apart from the Hořava-Lifshitz model, conclusions of the present work hold also for the mimetic matter scenario, where the analogue of the projectability condition is achieved by a non-invertible conformal transformation of the metric.

KEYwORDS: Models of Quantum Gravity, Cosmology of Theories beyond the SM, Gauge Symmetry

ARXIV EPRINT: 1601.05405 


\section{Contents}

1 Introduction and summary 1

2 Review of projectable Hor̆ava-Lifshitz gravity 4

2.1 Setup 4

$\begin{array}{lll}2.2 & \text { Low-energy quadratic action } & 7\end{array}$

2.3 Cubic interactions: determining the strong coupling scale 11

3 Vacuum decay $\quad 14$

3.1 Vacuum decay into photons from the 4-point contact interaction with ghosts 17

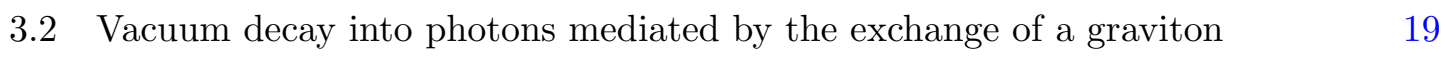

4 Discussion $\quad 21$

A On the equivalence between the IR limit of projectable Hořava-Lifshitz $\begin{array}{lr}\text { gravity and mimetic matter scenario } & 22\end{array}$

B Curing ghost instabilities beyond projectable Hořava-Lifshitz gravity 24

\section{Introduction and summary}

Modifications of gravity are the subject of intensive debate in the scientific community. The main interest comes from cosmology. In particular, we do not know yet the origin of $95 \%$ of the energy density in the Universe. While the Dark Matter component $(27 \%)$ can be relatively easily explained by an extension of the Standard Model, no convincing model of Dark Energy (68\%) has been proposed yet [1]. Besides these cosmological considerations, finding a viable modification of General Relativity (GR) is, in a certain sense, a matter of principle. The first attempts to promote the graviton to a massive field (the renowned Fierz-Pauli model, [2]), have been made well before the discovery of Dark Matter and Dark Energy. It turned out, however, that the Fierz-Pauli model is plagued by ghost instabilities [3] and an unacceptably low scale of the strong coupling [4]. Motivated by those problems, several proposals on Lorentz-invariant models of gravity have been put forward recently $[5,6]$.

Another interesting approach to modifying gravity is to break Lorentz-invariance spontaneously or explicitly. (See the review [7], the references therein and refs. [8-10]). This is argued to be a viable way of getting around the troubles with ghost instabilities and a low scale of the strong coupling [11-13]. Moreover, relaxing the Lorentz symmetry, one opens up an intriguing opportunity to construct Dark Energy, inflation and Dark Matter, with a row of interesting models exemplified in the ghost condensate context [14-16]. 
Violation of Lorentz invariance at the high energies may have dramatic consequences in a view of another long-standing problem - the renormalization of gravity. Following Hořava's proposal [17-19], one assigns different scaling dimensions to the time, $t$, and spatial coordinates, $\mathbf{x}$, in the ultraviolet (UV),

$$
\mathbf{x} \rightarrow b^{-1} \mathbf{x}, \quad t \rightarrow b^{-z} t,
$$

where $z$ is the so-called critical exponent. The scaling (1.1) fixes the high energy dispersion relation for the graviton to be of the form $\omega^{2} \propto \mathbf{p}^{2 z}$, where $\omega$ denotes the energy and $\mathbf{p}$ the momentum. Consequently, the behaviour of the propagators changes in the UV, potentially eliminating the divergence of the loop integrals. Power-counting (super-)renormalizable gravity corresponds to the choice $z=3(z>3)$. See the discussion in subsection 2.1.

However, breaking the Lorentz symmetry does not come at zero price. It implies the smaller group of diffeormorphisms compared to that of GR. As an immediate consequence, one has extra degrees of freedom, on top of the standard helicity-2 graviton. In particular, the lapse function, $N(t, \mathbf{x})$, is a dynamical variable in Hořava-Lifshitz gravity. This new degree of freedom suffers from gradient instabilities and furthermore leads to a very low strong coupling scale in the theory [20,21]. Looking for the solution of the problem, one either turns to extensions of the Hořava-Lifshitz gravity or imposes some conditions capable of eliminating the pathological mode. The first line of research was developed in ref. [22], and we do not touch it in the present paper. We follow another approach, proposed already in the original paper by Hořava [17], which is to impose the projectability condition.

In the projectable Hořava-Lifshitz gravity one entertains the possibility that the lapse $N$ is a function of time only, i.e., $N=N(t)$. The reason why this scenario is important is twofold. First, it is the only version of the Horrava-Lifshitz gravity which has been renormalized so far [26]. ${ }^{1}$ Second, it has quite a rich phenomenology. In particular, this model provides a candidate for the Dark Matter and an alternative to inflation [27-31].

Still, the model of interest appears to be problematic in the infrared limit (IR). At low spatial momenta, it is equivalent to GR plus a fluid characterized by the constant sound speed $c_{s}[21,23,25]$, - a statement, which becomes manifest upon performing the Stuckelberg trick $[21,23,24]$ (see subsection 2.2). In what follows, we will consider two branches of the projectable Hořava-Lifshitz gravity depending on the sign of the quantity $c_{s}^{2}$. The branch with the negative sound speed squared, i.e., $c_{s}^{2}<0$, is the only studied in the literature. It is plagued by gradient instabilities in the IR, which can be cured in the UV by the Lorentz-violating operators inherent in Hořava-Lifshitz gravity. Naively, by tuning the sound speed $c_{s}$ to be arbitrarily small, one could avoid any conflict with observations. This expectation, however, is not met in reality: the strong coupling scale is finite and depends crucially on the sound speed [23, 25],

$$
\Lambda_{p} \sim M_{\mathrm{Pl}} \cdot\left|c_{s}\right|^{3 / 2} .
$$

Here $M_{\mathrm{Pl}}$ denotes the Planck mass defined as $M_{\mathrm{Pl}} \equiv G^{-1 / 2}$, where $G$ is Newton's constant. Note that the cutoff (1.2) is applied to the spatial momenta $p \equiv|\mathbf{p}|$, i.e., it breaks Lorentz

\footnotetext{
${ }^{1}$ However, in 4 dimensions the perturbative renormalization of the projectable Hořava-Lifshitz gravity is achieved at the price of the phenomenological viability of the model. See the discussion below.
} 
invariance explicitly. Demanding that gradient instabilities do not propagate on a time scale smaller than the age of the Universe, leads to the constraint $\Lambda_{p} \lesssim 10^{-17} \mathrm{eV}$ [23]. This is by many orders of magnitude below the phenomenologically allowed values.

In this paper, we will argue that the second branch, with $c_{s}^{2}>0$, of the Hořava-Lifshitz gravity is viable in a much wider range of sound speed values, so that the model remains perturbative down to microscopic scales. The reason, why this branch has been ignored in the literature is simple: unlike standard cosmological fluids, e.g., radiation, in our case the positive sound speed squared comes at the price of having ghost instabilities [22, 23, 25]. Naively, this invalidates the scenario of interest. However, it is well-known that ghosts are not particularly dangerous in Lorentz-violating theories of gravity [12]. ${ }^{2}$ That is, for a reasonably small cutoff $\Lambda$ on the momenta and the frequencies of the ghosts, the decay of the vacuum into Standard Model species in the final state is sufficiently slow, - in agreement with the observed abundance of particles in the Universe. ${ }^{3}$ In the projectable Hořava-Lifshitz gravity that cutoff is realized by the finite scale of the strong coupling (1.2), above which, we assume, the model is free of instabilities. The upper limit on the scale $\Lambda$ obtained in ref. [12] reads $\Lambda \lesssim 3 \mathrm{MeV}$. The latter is applied provided that the frequency $\omega$ and the spatial momenta $\mathbf{p}$ have equal cutoffs. This condition, however, is not fulfilled in our scenario, where the ghosts obey the non-relativistic dispersion relation $\omega^{2}=c_{s}^{2} \mathbf{p}^{2}$, with the sound speed much less than unity, i.e., $c_{s} \ll 1$. As a by-product, the cutoff on the spatial momenta (strong coupling scale (1.2)) can be relaxed by many orders of magnitude, extending the validity of perturbation theory to the $\mathrm{TeV}$ range (see section 3 ).

The reason is twofold. First, in the formal limit $c_{s} \rightarrow 0$, the energies of the ghosts and, say, the photons produced from the vacuum decay approach zero. This leads to a vanishing phase-space volume of the outgoing particles. For generic values of the sound speed (still, much smaller than unity), this argument implies the suppression of the phase-space volume by some power of the quantity $c_{s}$. Second, the coupling between the standard fields, i.e., photons and helicity-2 gravitons, with the canonical ghost field is very weak. This follows from normalization considerations. Collecting the suppressing factors altogether and comparing the resulting decay rate into photons with the observed flux of cosmic Xrays, one obtains $c_{s}^{2} \lesssim 10^{-20}$. The upper limit here implies that the strong coupling scale in the projectable Hořava-Lifshitz gravity can be as large as $\Lambda_{p} \sim 10 \mathrm{TeV}$. This is about thirty orders of magnitude weaker than the associated limit in the branch plagued by the gradient instabilities.

To summarize, the branch of the projectable Hořava-Lifshitz gravity with the ghosts in the IR is a phenomenologically viable scenario. We note, however, that the presence of the strong coupling by itself is not good from the viewpoint of renormalizing HořavaLifshitz gravity. ${ }^{4}$ Naively, one could circumvent the problem by setting the UV cutoff of the theory somewhat below the would be strong coupling scale. In that case, the computation

\footnotetext{
${ }^{2}$ The opportunity to have controllable ghost instabilities in Lorentz-invariant theories is discussed in ref. [32].

${ }^{3}$ See refs. [33] and [34] for the applications of this idea to various gravitational frameworks.

${ }^{4}$ In particular, the presence of the strong coupling invalidates the discussion of ref. [26], where it is assumed that the model can be treated perturbatively at the arbitrary scale.
} 
of the strong coupling cutoff performed within the IR theory is not valid. Instead, one can argue that the theory remains perturbative at an arbitrary scale [35]. However, this is not a viable option in the branch with the positive sound speed squared. The reason is that the ghost instabilities are not cured by the operators arising in the UV. Hence, retaining perturbativity would make them propagate at an arbitrary scale and lead to an instantaneous destabilization of the vacuum. Thus, we are forced to assume that the UV cutoff is above the strong coupling scale, and hence the projectable Hořava-Lifshitz gravity enters the genuinely non-perturbative regime at some point. This is a non-appealing feature of the IR theory, which severely obscures its UV completion. We sketch a possible solution of the problem in the end of subsection 2.2 and in appendix. This solution, however, involves operators beyond the projectable Horava-Lifshitz gravity and, thus, is out of the main scope of the paper.

Apart from the issues with the UV completion, the scenario at hand looks attractive from a somewhat more down-to-earth perspective: it allows us to construct a fluid free of any obvious pathologies characterized by a small sound speed. In particular, that fluid could be an interesting candidate for Dark Matter. While practically indistinguishable from a collection of cold non-interacting particles at the background and linear levels [27, 36-39], it exhibits a different behaviour in the non-linear phase [31, 38]. These features might be of sufficient interest in view of the series of small scale problems alleged to the Cold Dark Matter [40].

Finally, we note that the results of the present paper can be literally translated into the context of the mimetic matter scenario [36]. The latter is a novel proposal, originally designed to explain the Dark Matter in the Universe by a singular disformal transformation of the metric in GR [41]. In fact, the mimetic matter scenario extended by means of a higher derivative term [37] is equivalent to the IR limit of the projectable Horava-Lifshitz gravity, as well as to a particular version of the Einstein-Aether theory [42-44]. The classical evolution of cosmological perturbations in that setup was studied in refs. [37, 38], and the stability issues have been partially addressed in ref. [45]. In a certain sense, the results of the present paper extend those analyses to the quantum level.

The paper is organized as follows. In section 2, we review the IR limit of the HoravaLifshitz gravity with the projectability condition imposed and re-derive the strong coupling scale. In section 3, we estimate the decay rate of the vacuum into photons and ghosts and establish an upper limit on the sound speed $c_{s}$ and, consequently, on the scale of the strong coupling. Finally in section 4 , we discuss some prospects for future research.

\section{Review of projectable Hořava-Lifshitz gravity}

\subsection{Setup}

We start with a brief review of the Hořava-Lifshitz gravity theory, focusing on its projectable version. We write the metric using the Arnowitt-Deser-Misner (ADM) formalism [46],

$$
d s^{2}=N^{2} d t^{2}-\gamma_{i j}\left(d x^{i}+N^{i} d t\right)\left(d x^{j}+N^{j} d t\right)
$$


(we assume the mostly negative signature). Along with eq. (1.1), one postulates the following scaling of the lapse function $N$, the shift vector $N_{i}$ and the metric on the constant $t$-hypersurface $\gamma_{i j}[17]$,

$$
\gamma_{i j} \rightarrow \gamma_{i j}, \quad N_{i} \rightarrow b^{2} N_{i}, \quad N \rightarrow N
$$

Here the value of the critical exponent $z=3$ is implied. Next, one classifies all the possible operators according to their scaling dimensions. In particular, the operators entering the GR kinetic term, i.e.,

$$
K_{i j} K^{i j}, \quad K^{2},
$$

have scaling dimension 6 . Here $K_{i j}$ and $K$ are the extrinsic curvature tensor and its trace, respectively. The operators of the same dimension as the kinetic terms are called marginal. The action of the Hořava-Lifshitz gravity is then designed as the sum of all marginal and relevant operators [17]. These can be assembled in the GR fashion,

$$
S=\frac{1}{16 \pi G} \int d t d^{3} x \sqrt{\gamma} N\left(K_{i j} K^{i j}-\lambda K^{2}-\mathcal{V}\right),
$$

where $\mathcal{V}$ is the so called potential typically involving powers of the 3-dimensional Ricci scalar and tensor as well as their derivatives [17, 47, 48]. Just to illustrate the main idea behind Hořava's proposal, let us write down an example of an operator entering the potential $\mathcal{V}$,

$$
\frac{1}{M_{*}^{4}} R \Delta R .
$$

Here $R$ is the Ricci scalar calculated on the constant $t$-hypersurface; $M_{*}$ is a free parameter defining the scale of the UV completion of the theory. In the presence of the marginal operator (2.2), the graviton propagator gets modified as follows [17],

$$
\frac{1}{\omega^{2}-\mathbf{k}^{2}} \rightarrow \frac{1}{\omega^{2}-\mathbf{k}^{2}-\frac{\left(\mathbf{k}^{2}\right)^{3}}{M_{*}^{4}}} .
$$

That is, at sufficiently long distances, one recovers the standard GR behaviour of the propagator. At high energies, the scaling of the propagator strongly improves. Consequently, one opens up the possibility to reduce the divergencies of the loop integrals. In particular, the behaviour as in eq. (2.3) leads to the power counting renormalizable gravity in 4 dimensions.

Having briefly described the UV properties of the Hořava-Lifshitz gravity, we switch to its IR limit, - the main focus of our studies. In that limit, the potential $\mathcal{V}$ takes the form, ${ }^{5}$

$$
\mathcal{V} \rightarrow-R
$$

Substituting this into the action (2.1), we obtain the action of GR, modulo the constant $\lambda$, which is generically not equal to unity. Naively, Einstein's gravity is recovered in the limit

\footnotetext{
${ }^{5}$ Generically, one should write $\mathcal{V} \rightarrow-\zeta R$, where $\zeta$ is some arbitrary constant. This, however, can be safely tuned to unity to better match the GR case.
} 
$\lambda \rightarrow 1$. However, this is not so. The reason is that the action of Hořava-Lifshitz gravity respects a smaller group of diffeomorphisms compared to that of GR [17],

$$
t \rightarrow \tilde{t}(t), \quad \mathbf{x} \rightarrow \tilde{\mathbf{x}}(t, \mathbf{x})
$$

called foliation-preserving diffeomorphisms. This eventually leads to an additional degree of freedom on top of the helicity- 2 graviton. The new degree of freedom typically exhibits a pathological behaviour in the IR or leads to the breakdown of perturbation theory at unacceptably large distances $[20,21]$. So, unlike in GR, in Hořava-Lifshitz gravity (or, at least, in its original incarnation) the lapse $N(t, \mathbf{x})$ is a dynamical variable. This variable is plagued by gradient instabilities and a very low strong coupling scale.

That problem is absent in the projectable version of the Horrava-Lifshitz gravity [17], to which we turn now. There, one assumes that the lapse is a function of time only, i.e., $N=N(t)$. In particular, it can be set to unity, i.e., $N(t)=1$, by the proper choice of the time reparametrization. To enforce the condition $N=1$, following ref. [22], we introduce a term in the action with a Lagrange multiplier $\Sigma$,

$$
S_{\mathrm{fix}}=\int d^{3} x d t \sqrt{\gamma} N \frac{\Sigma}{2}\left(\frac{1}{N^{2}}-1\right) .
$$

This is not the only option. Instead, one may consider a ghost condensate-like term [23], i.e., $M^{4}\left(1 / N^{2}-1\right)^{2}$, where $M$ is a fictitious mass parameter. The projectability condition is then ensured by imposing the limit $M \rightarrow \infty$. In what follows, we will see that the two strategies lead to the same conclusions. Combining eqs. (2.1), (2.4) and (2.5), we are ready to write down the action for the IR limit of the projectable Hořava-Lifshitz gravity,

$$
S_{\mathrm{IR}}=S_{\mathrm{GR}}+\int d^{3} x d t \sqrt{\gamma} N\left[\frac{\Sigma}{2}\left(\frac{1}{N^{2}}-1\right)-\frac{\lambda-1}{16 \pi G} K^{2}\right] .
$$

We explicitly grouped a part of the terms into the GR action $S_{\mathrm{GR}}$.

Despite the absence of the problems with the lapse, the model (2.6) has a propagating helicity-0 mode, which exhibits ghost or gradient instabilities depending on the value of the parameter $\lambda[23,25]$. To study the properties of that mode is the main goal of the present paper. For this purpose, the action (2.6) is not very convenient. Instead, we choose to work with an equivalent formulation of eq. (2.6), which is manifestly invariant under the full GR diffeomorphisms. This is achieved by performing the Stuckelberg trick, with the Stuckelberg field $\varphi$ dubbed as khronon in the context of the Hořava-Lifshitz gravity [21$24,49]$. Note that by introducing the field $\varphi$, one does not enlarge the number of degrees of freedom in the theory, but singles out those already present. In particular, the dynamics of the field $\varphi$ is eliminated by imposing the unitary gauge, where it takes the form,

$$
\varphi=t
$$

We see that the field $\varphi$ defines the absolute time. Hence, the name 'khronon'.

To write the action (2.6) in covariant form, it is enough to promote the lapse $N$ and the extrinsic curvature $K_{\mu \nu}$ to the quantities invariant under GR diffeomorphisms. This 
has been done in ref. [21], and we omit the details of the calculations here. The result for the lapse $N$ reads,

$$
\frac{1}{N^{2}} \rightarrow g^{\mu \nu} \partial_{\mu} \varphi \partial_{\nu} \varphi
$$

Now $K_{\mu \nu}$ is the extrinsic curvature of the hypersurface $\varphi=$ const, and its trace is given by

$$
K \rightarrow \nabla_{\mu}\left(\frac{\nabla^{\mu} \varphi}{\sqrt{g^{\mu \nu} \partial_{\mu} \varphi \partial_{\nu} \varphi}}\right) .
$$

This expression can be simplified by virtue of the constraint enforced by the Lagrange multiplier $\Sigma$. In the Stuckelberg treatment the constraint is given by,

$$
g^{\mu \nu} \partial_{\mu} \varphi \partial_{\nu} \varphi=1
$$

This can be safely plugged into eq. (2.8) readily at the level of the action. See appendix A for the justification. As a result, eq. (2.8) gets simplified,

$$
K \rightarrow \square \varphi .
$$

The covariant action for the IR limit of the projectable Hořava-Lifshitz gravity is given by [23],

$$
S_{\mathrm{IR}}=S_{\mathrm{GR}}+\int d^{4} x \sqrt{-g}\left[\frac{\Sigma}{2}\left(g^{\mu \nu} \partial_{\mu} \varphi \partial_{\nu} \varphi-1\right)+\frac{\gamma}{2}(\square \varphi)^{2}\right],
$$

where we introduced the shorthand notation,

$$
\frac{1-\lambda}{8 \pi G}=\gamma
$$

In passing, it is interesting to note that the action (2.10) by itself is not specific to the projectable Hořava-Lifshitz gravity, but may arise in a drastically different framework. In this regard, the mimetic matter scenario has brought some attention recently [36]. The idea is to consider a particular (singular) conformal transformation of the metric, i.e., $g_{\mu \nu} \rightarrow \tilde{g}_{\mu \nu}=g^{\alpha \beta} \partial_{\alpha} \varphi \partial_{\beta} \varphi \cdot g_{\mu \nu}$. Here $\varphi$ is some scalar field. This transformation does not leave the GR equations of motion invariant. The discrepancy from GR is equivalent to extending the Einstein-Hilbert action by means of the term with the Lagrange multiplier as in eq. (2.10) $[45,50,51]$. There is, however, a conceptual distinction from the HořavaLifshitz model. In the mimetic matter case, the higher derivative term as in eq. (2.10) is added in view of some phenomenological goals [37-39], i.e., it does not follow immediately from the first principles underlying the scenario.

Keeping in mind this potentially interesting scenario, we proceed with the HoraavaLifshitz model as the main focus of the present work. The action (2.10) will be the starting point of our further discussions.

\subsection{Low-energy quadratic action}

Interestingly, at the level of the background cosmological equations, the model given by the action (2.10) describes dust (pressureless perfect fluid). This readily follows from the $i j$-components of Einstein's equations [38, 39, 44],

$$
2 \mathcal{H}^{\prime}+\mathcal{H}^{2}=0
$$


The 00-component of Einstein's equations is given by

$$
3 \mathcal{H}^{2}(1-24 \pi \gamma G)=8 \pi G a^{2} \bar{\Sigma}
$$

Here a prime denotes the derivative with respect to the conformal time, $\mathcal{H} \equiv a^{\prime} / a, a$ is the scale factor, and $\bar{\Sigma}$ is the background value of the Lagrange multiplier field. As it follows from eqs. (2.12) and (2.13), at the background level the higher derivative term is irrelevant: effects due to the non-zero parameter $\gamma$ can be absorbed into the redefinition of the cosmological Newton's constant. That degeneracy with the case of the pressureless fluid gets broken at the linear level, where the $\gamma$-term gives rise to a non-zero scalar sound speed as will be clear shortly. Before that, let us point out an intriguing possibility to mimic the energy density of the Dark Matter by the field $\Sigma$, i.e., without invoking physics beyond the Standard Model of particles. In this picture, the khronon $\varphi$ is understood as the velocity potential, while the constraint (2.9) leads to the geodesics equation followed by the dust particles in the gravitational field. The properties of the Dark Matter in the model (2.10) and closely related scenarios have been explored in refs. [27, 31] in the context of the projectable Hořava-Lifshitz gravity, in refs. [36-39, 45, 50-53] in the mimetic matter setup and in ref. [54] on purely phenomenological grounds. ${ }^{6}$ That line of research is out of the scope of the present paper.

Naively, the higher derivative term in the action (2.10) would lead to an Ostrogradski instability. However, the Ostrogradski theorem is not applicable to models with constraints [57-59] (the so-called degenerate systems). The reason is that the addition of the constraints may reduce the dimension of the Hamiltonian phase space, and consequently, the number of the propagating degrees of freedom may be smaller compared to the naive counting. This is indeed the case in the projectable Hořava-Lifshitz gravity. In particular, the scalar sector of the model has only one propagating degree of freedom.

To study linear perturbations in the projectable Hořava-Lifshitz model, we use the standard conventions [60,61],

$$
N=a(1+\Phi), \quad N_{i}=a^{2} \partial_{i} B, \quad \gamma_{i j}=a^{2}(1-2 \Psi) \delta_{i j}+2 a^{2} \partial_{i} \partial_{j} E
$$

Here $\Phi, \Psi, B, E$ are the scalar potentials, and we omit the discussion of the vector and tensor modes (see the comment at the end of this subsection).

At the linear level, the constraint $g^{\mu \nu} \partial_{\mu} \varphi \partial_{\nu} \varphi=1$ gives

$$
\frac{\delta \varphi^{\prime}}{a}=\Phi
$$

The latter is enforced by the Lagrange multiplier field $\Sigma$. Therefore, one can safely use eq. (2.14) to eliminate the field $\Phi$ without affecting the dynamics of the remaining degrees of freedom [63]. Upon substituting eq. (2.14) in the quadratic action, integrating by parts,

\footnotetext{
${ }^{6}$ Note that Dark Matter is the prediction specific to the projectable version of the Hořava-Lifshitz model. Still, in the different extensions of the model, one can entertain the opportunity of having MOND-like phenomenology in the IR limit $[55,56]$.
} 
dropping boundary terms and making use of the background equations (2.12) and (2.13), we obtain

$$
\begin{aligned}
\delta_{2} S_{\mathrm{IR}}= & \frac{1}{16 \pi G} \int d^{4} x a^{2}\left[-6 \mathcal{R}^{\prime 2}+2 \Delta \Psi\left(2 \frac{\delta \varphi^{\prime}}{a}-\Psi\right)+\frac{3 \mathcal{H}^{2} \delta \varphi \Delta \delta \varphi}{a^{2}}+4 \mathcal{R}^{\prime} \cdot\left(\Delta E^{\prime}-\Delta B\right)\right]+ \\
& +\frac{\gamma}{2} \int d^{4} x a^{2}\left(3 \mathcal{R}^{\prime}+\frac{\Delta \delta \varphi}{a}+\Delta B-\Delta E^{\prime}\right)^{2} .
\end{aligned}
$$

Here $\mathcal{R}$ is the gauge-invariant curvature perturbation, defined as $\mathcal{R}=\Psi+\frac{\mathcal{H}}{a} \delta \varphi$. Note that the action (2.15) does not assume any gauge choice. The fields $B$ and $E$ enter only via the combination $B-E^{\prime}$, as it should be, because of diffeomorphism invariance. The variation with respect to the field $B$ (or $E^{\prime}$ ) yields

$$
\Delta B-\Delta E^{\prime}=\frac{1}{4 \pi \gamma G} \mathcal{R}^{\prime}-3 \mathcal{R}^{\prime}-\frac{\Delta \delta \varphi}{a} .
$$

At this level, we explicitly assume that the parameter $\gamma$ is non-zero, i.e., $\gamma \neq 0$. As it follows from eq. (2.16), $B$ is an auxiliary field. Namely, it is separated from the other fields by means of its own equation of motion. Therefore, one can safely substitute the constraint (2.16) into the quadratic action (2.15) [63]. Doing so, we obtain

$$
\delta_{2} S_{\mathrm{IR}}=\frac{1}{8 \pi G} \int d^{4} x a^{2}\left(-\frac{1}{4 \pi \gamma G} \mathcal{R}^{\prime 2}+3 \mathcal{R}^{\prime 2}-\mathcal{R} \Delta \mathcal{R}\right) .
$$

As it was expected, there is only one propagating degree of freedom in the scalar sector of the model. Furthermore, in the unitary gauge $\delta \varphi=0$, one reproduces the result of ref. [23]. This serves as a simple cross-check of our calculations.

Note that the action (2.17) has a continuous limit to the flat space-time. This allows us to set consistently $a \rightarrow 1$ and $\mathcal{H} \rightarrow 0$, and ignore the effects related to the expansion of the Universe in what follows. These have been already discussed to some extent in refs. [27, 36-39, 54]. In the Minkowski limit, the gauge-invariant variable $\mathcal{R}$ takes the form $\mathcal{R}=\Psi$, and the action (2.17) reduces to,

$$
\delta_{2} S_{\mathrm{IR}}=\frac{1}{8 \pi G} \int d^{4} x\left(-\frac{1}{c_{s}^{2}} \dot{\Psi}^{2}-\Psi \Delta \Psi\right) .
$$

Here $c_{s}^{2}$ is the sound speed squared given by [22, 23, 25],

$$
c_{s}^{2}=\frac{4 \pi \gamma G}{1-12 \pi \gamma G} .
$$

As it follows, the IR properties of the field $\Psi$ are characterized by the phonon-like dispersion relation

$$
\omega^{2}=c_{s}^{2} \mathbf{p}^{2} .
$$

Hereafter, we assume the hierarchy $\gamma \ll M_{\mathrm{Pl}}^{2}$, which allows us to simplify the expression for the sound speed squared $c_{s}^{2}$,

$$
c_{s}^{2}=4 \pi \gamma G .
$$


This is, in fact, the only phenomenologically viable option, as it will be clear from the following discussions.

Let us start with the case $\gamma=0$. This corresponds to GR supplemented by a pressureless perfect fluid (dust), which is a classically well-defined system (up to the caustic singularities). On the other hand, the action (2.18) is ill-defined in that case. However, this does not signal the inconsistency in the discussion, as we assumed the choice $\gamma \neq 0$ at the intermediate step (see the comment after eq. (2.16)). ${ }^{7}$ One obvious way to handle the situation is to consider the limit of the infinitely small parameter $\gamma$, i.e., $\gamma \rightarrow 0$, instead of setting it exactly to zero. Then, demanding that the action $(2.18)((2.17))$ remains finite, one obtains the first order equation for the potential $\Psi$ (the curvature perturbation $\mathcal{R}$ ): $\dot{\Psi}=0\left(\mathcal{R}^{\prime}=0\right)$. The latter is recognized as the conservation of the potential (curvature perturbation) characteristic of dust. There is a more trustworthy way to get the same equation. That is, one gets back to the original quadratic action (2.15), which is manifestly applicable for the arbitrary values of the parameter $\gamma$. Then, the conservation of the curvature perturbation follows immediately upon varying with respect to the field $B$. Furthermore, one may check that all the other equations following from eq. (2.15) (with $\gamma=0$ understood) are the same as in GR supplemented by a pressureless perfect fluid. Although the simple dust model is typically employed to describe the behaviour of Dark Matter on cosmological scales, it has two important drawbacks rendering the case $\gamma \rightarrow 0$ pathological. First, the quantum properties of the model are unclear, as the strong coupling scale tends to zero in that limit $[23,25] .{ }^{8}$ See the discussion in the next subsection. Second, a pressureless perfect fluid develops caustic singularities at a finite time [62].

Therefore, we switch to the case $\gamma \neq 0$ in what follows. For negative values of the parameter $\gamma$ (sound speed squared), the field $\Psi$ suffers from gradient instabilities. On the other hand, positive values of the parameter $\gamma$ (sound speed squared), lead to the 'wrong' sign of the kinetic term in the action (2.18). The study of this ghost-unstable branch of the projectable Hořava-Lifshitz gravity will be our primary interest in the present paper.

Note one important difference between ghost and gradient instabilities in the projectable Hořava-Lifshitz gravity. At sufficiently high spatial momenta, the action (2.18) must be completed by the relevant and marginal operators encoded in the potential $\mathcal{V}$. In the unitary gauge, these result into quadratic terms of the form,

$$
\frac{1}{M_{*}^{2}} \Psi \Delta^{2} \Psi, \quad \frac{1}{M_{*}^{4}} \Psi \Delta^{3} \Psi
$$

Recall that $M_{*}$ is the scale at which the Lorentz-violating operators become important presumably renormalizing gravity in the UV. As it follows from the structure of the terms (2.21), they are capable to cure gradient instabilities in the UV, while leaving the sign of the kinetic term in eq. (2.18) intact. Hence, from the higher derivative perspective alone, ghosts are unavoidable in the scenario with the positive parameter $\gamma$. This situation

\footnotetext{
${ }^{7}$ Of course, one can rewrite eq. (2.16) in the form applicable for both cases $\gamma=0$ and $\gamma \neq 0$, i.e., $\gamma\left(\Delta B-\Delta E^{\prime}\right)=\frac{1}{4 \pi G} \mathcal{R}^{\prime}-3 \gamma \mathcal{R}^{\prime}-\gamma \frac{\Delta \delta \varphi}{a}$. Still, the choice $\gamma=0$ is 'singular' in a sense that it does not allow to integrate out the field $B$, which plays the role of the Lagrange multiplier now.

${ }^{8}$ Alternatively, the problem with the quantization can be understood from the fact that the curvature perturbation satisfies the first order equation of motion in the limit $c_{s} \rightarrow 0$.
} 
may change due to the presence of sufficiently low strong coupling scale, above which the theory is hopefully free of ghost/gradient instabilities.

This problem with the ghosts, we note, is specific to the projectable Hořava-Lifshitz model, and can be avoided by relaxing some assumptions underlying the framework. Here is a sketch of one possible solution. Let us assume an extension of the Horava-Lifshitz gravity by means of the operator,

$$
\frac{1}{M_{*}^{2}} K \Delta K
$$

Recall that $K$ is the trace of the extrinsic curvature tensor. Generically, the operators of the form (2.22) may compromise power counting renormalizability of the Horava-Lifshitz gravity [64-66]. Therefore, they have been omitted in the original action (2.1). On the other hand, the term (2.22) and similar ones are quite well motivated, as they allow to stabilize the percolation of the Lorentz-violating effects from the gravity sector to the particle one [67].

In the Stuckelberg treatment, the operator (2.22) can be rewritten as follows

$$
\frac{1}{M_{*}^{2}} \square \varphi\left(\square-\partial_{\mu} \varphi \partial_{\nu} \varphi \nabla^{\mu} \nabla^{\nu}\right) \square \varphi
$$

Introducing this term into the action (2.10) indeed allows us to recover the positive sign of the kinetic term of the potential $\Psi$ in the UV, i.e., for spatial momenta $|\mathbf{p}| \gtrsim M_{*}$. We relegate the details of the computations to the appendix $\mathrm{B}$, and postpone a more thorough analysis for future work.

One comment is in order here. Compared to the scalar sector, the tensor part of the projectable Hořava-Lifshitz gravity exhibits healthy behaviour in the IR limit. Namely, it is free of ghost/gradient instabilities and the strong coupling issues. See, e.g., eq. (9) of ref. [26]. Nevertheless, the interaction with the strongly coupled scalar sector may essentially affect the behaviour of the tensor modes at larger momenta, severely obscuring their UV properties.

\subsection{Cubic interactions: determining the strong coupling scale}

The strong coupling scale has been calculated previously in refs. [23, 25] and is given by eq. (1.2). In the present subsection, we re-derive this result by employing the Newtonian gauge.

To understand the structure of the cubic interactions, we expand the constraint equation (2.14) up to the quadratic terms in the potential $\Phi$ and the khronon field perturbations $\delta \varphi,{ }^{9}$

$$
\Phi=\delta \dot{\varphi}+\frac{1}{2} \delta \dot{\varphi} \delta \dot{\varphi}-\frac{1}{2} \partial_{i} \delta \varphi \partial_{i} \delta \varphi .
$$

Note that the terms $\sim \Phi^{2}$ and $\sim \delta \dot{\varphi} \cdot \Phi$ cancel out upon implementing the first order constraint $\delta \dot{\varphi}=\Phi$. In the Newtonian gauge, the strong coupling stems from the following term in the GR action,

$$
\sim M_{\mathrm{Pl}}^{2} \Delta \Psi \Phi,
$$

\footnotetext{
${ }^{9}$ Recall that we choose to work in the Minkowski background.
} 
(cf. the second term in the first line of eq. (2.15)). This is quadratic in the fields $\Psi$ and $\Phi$, and, thus, naively does not correspond to any interaction. According to eq. (2.24), however, the potential $\Phi$ is sourced by the quadratic order perturbations in the khronon field. Therefore, we are left with the following interaction,

$$
\sim M_{\mathrm{Pl}}^{2} \Delta \Psi \partial_{i} \delta \varphi \partial_{i} \delta \varphi
$$

We ignored the terms with the time derivatives in eq. (2.24). This is legitimate in view of the dispersion relation $\omega^{2}=c_{s}^{2} \mathbf{p}^{2}$, where $c_{s}^{2} \ll 1$. The field $\delta \varphi$ is extracted from the constraint (2.16), where we set the fields $B$ and $E$ to zero by the Newtonian gauge choice,

$$
\delta \varphi \sim \frac{M_{\mathrm{Pl}}^{2} \dot{\Psi}}{\gamma \Delta} .
$$

Substituting this into eq. (2.26), we get an estimate for the cubic interaction,

$$
\sim \frac{M_{\mathrm{Pl}}^{6} \Psi}{\gamma^{2} \Delta}\left(\partial_{i} \dot{\Psi}\right)^{2}
$$

Again, taking into account the dispersion relation (2.19) and eq. (2.20), one rewrites the estimate above as follows,

$$
\sim \frac{M_{\mathrm{Pl}}^{4}}{\gamma} \Psi\left(\partial_{i} \Psi\right)^{2}
$$

This is to be compared with the standard quadratic term in the GR action involving the spatial derivatives of the potential $\Psi,{ }^{10}$

$$
\frac{\frac{M_{\mathrm{Pl}}^{4}}{\gamma} \Psi\left(\partial_{i} \Psi\right)^{2}}{M_{\mathrm{Pl}}^{2} \Psi \Delta \Psi} \sim \frac{M_{\mathrm{Pl}}^{2}}{\gamma} \Psi .
$$

To proceed, we need an estimate for the amplitude of the fluctuations of the field $\Psi$. For this purpose, we switch to the canonical normalized variable $\hat{\Psi}=\frac{M_{\mathrm{Pl}}^{2}}{\sqrt{\gamma}} \Psi$. Fluctuations of the variable $\hat{\Psi}$ are characterized by a Gaussian distribution with zero mean value and the variance,

$$
\left\langle\hat{\Psi}^{2}\right\rangle \sim \int \frac{d \mathbf{p}}{|\omega(\mathbf{p})|} \sim \frac{M_{\mathrm{Pl}}}{|\gamma|^{1 / 2}}|\mathbf{p}|^{2} .
$$

Returning to the variable $\Psi$, we obtain the estimate for its fluctuations,

$$
\Psi \sim \sqrt{\left\langle\Psi^{2}\right\rangle} \sim \frac{|\gamma|^{1 / 4}|\mathbf{p}|}{M_{\mathrm{Pl}}^{3 / 2}} .
$$

Combining everything together and demanding that the ratio (2.27) does not exceed unity, we conclude that the scale of the strong coupling is

$$
\Lambda_{p} \sim \frac{|\gamma|^{3 / 4}}{M_{\mathrm{Pl}}^{1 / 2}} .
$$

\footnotetext{
${ }^{10}$ More rigorously, to deduce the strong coupling scale, one calculates the cross-section of the scattering of two $\Psi$-particles. The obtained cross-section should not violate the optical theorem, i.e., unitary must be obeyed. This gives a constraint on the allowed values of the momenta. We followed this way, and showed that the result matches the one given below.
} 
This result exactly matches the one obtained in refs. [23, 25]. In particular, the scale of strong coupling tends to zero in two limits: in the decoupling limit $\left(M_{\mathrm{Pl}} \rightarrow \infty\right)$ and in the limit of the pressureless perfect fluid $(\gamma \rightarrow 0)$. Note that the cutoff $(2.28)$ is applied to the spatial momenta only, i.e., it breaks Lorentz-invariance explicitly. Hence, the subscript ' $p$ '. To understand the region of energies which can be treated perturbatively, one simply makes use of the dispersion relation (2.19). This yields,

$$
\Lambda_{\omega} \sim \frac{|\gamma|^{5 / 4}}{M_{\mathrm{Pl}}^{3 / 2}} .
$$

That cutoff is not of particular importance in the projectable Hořava-Lifshitz gravity, but merely reflects the fact that the quanta of the field $\Psi$ are 'slow'. Indeed, the UV completing operators written schematically in eq. (2.21) carry only spatial derivatives, and thus, are sensitive only to the scale (2.28).

For the model to be phenomenologically viable, the strong coupling scale $\Lambda_{p}$ must be larger than the maximal scale, at which GR has been tested, i.e.,

$$
\Lambda_{p} \gtrsim 10^{-3} \mathrm{eV}
$$

This translates into the bound on the parameter $\sqrt{|\gamma|}$,

$$
\sqrt{|\gamma|} \gtrsim 10 \mathrm{MeV}
$$

In the branch of the projectable Hořava-Lifshitz gravity plagued by the gradient instabilities at low spatial momenta, the associated constraints are orders of magnitude stronger [23], making it phenomenologically non-viable. Let us show this explicitly. Due to the presence of the Lorentz-violating terms as in eq. (2.21), gradient instabilities are cut at the scale $|\mathbf{p}| \sim M_{*}$. One then demands that the time at which they propagate exceeds the age of the Universe. Namely, $|\operatorname{Im} \omega| \lesssim\left|c_{s}\right| M_{*} \lesssim H_{0}$, where $H_{0}$ is the Hubble constant. The parameter $M_{*}$ is bounded from below, $M_{*} \gtrsim 10^{-3} \mathrm{eV}$, - otherwise, Lorentz violating effects would pop out at sub-mm scales, in conflict with GR tests. Combining everything together, we obtain the constraint on the strong coupling scale: $\Lambda_{p} \lesssim 10^{-17} \mathrm{eV}$ [23]. This is by many orders of magnitude lower than the allowed value (2.30). Strictly speaking, having the hierarchy $\Lambda_{p} \ll M_{*}$, one cannot trust these results, as they were obtained by exploiting the region of the momenta, where perturbation theory breaks down. Instead, let us assume that gradient instabilities are cut by the scale of the strong coupling itself - with the hope that the theory is free of any instabilities in the non-linear regime. In that case, the range of the momenta, where the theory can be treated perturbatively, is slightly extended: $\Lambda_{p} \lesssim 10^{-8} \mathrm{eV},-$ still in conflict with the GR tests.

From this point on, we abandon the branch of the Hořava-Lifshitz gravity characterized by the negative sound speed squared, and switch to the one plagued by the ghosts in the IR. In the end of the previous subsection, we observed that the ghosts cannot be cured in the UV, at least in the projectable Hořava-Lifshitz gravity, as it stands. In this regard, the presence of the strong coupling is necessary to render the model phenomenologically acceptable. Namely, above the scale $\Lambda_{p}$, the results of the linear theory are not valid 
anymore. Therefore, we may assume that the model is free of instabilities in the nonperturbative regime. While looking quite speculative, this expectation has some reasons behind it. Indeed, the term in the GR action, which is responsible for the strong coupling, in the linear theory gives rise to the kinetic term with the negative sign (see the second term in eq. (2.15)).

Accordingly to the discussion above, we must set the UV cutoff $M_{*}$ somewhat higher than the scale $\Lambda_{p}$,

$$
M_{*} \gtrsim \Lambda_{p}
$$

Otherwise, our conclusions about the scale of the strong coupling would not be legitimate. Indeed, the estimate (2.28) has been obtained within the IR theory, and must be revisited, if the UV operators (2.21) become relevant already in the weakly coupled regime. In that case, one can argue that the model retains perturbativity at the arbitrary momenta [35]. This inevitably causes the presence of the all-scale ghost instabilities, and, consequently, a catastrophically fast vacuum decay.

To summarize, by imposing the condition (2.32), we sacrifice the renormalization of the Hořava-Lifshitz gravity in favour of its phenomenological viability. Let us be not too pessimistic, however. Indeed, apart from the projectable Hořava-Lifshitz model, the two issues, - low scale ghosts and strong coupling at large momenta, - are not necessarily related to each other. In particular, introducing the higher dimension operators, as, e. g., in eq. (2.22), one can simultaneously recover the positive sign of the kinetic term of the potential $\Psi$, and retain perturbativity of the theory. In the current work our goal is modest - to show that the projectable Hořava-Lifshitz gravity in its original incarnation is experimentally acceptable in the branch containing ghosts. Therefore, we postpone any detailed investigation of this potentially interesting loophole for the future.

\section{Vacuum decay}

Now, let us consider vacuum decay into ghosts and Standard Model particles. In the Lorentz-invariant theories of gravity, the associated decay rate is infinite. ${ }^{11}$ The situation is different in the Lorentz-violating theories, given that there is a low energy cutoff $\Lambda$ on the spatial momenta [12]. ${ }^{12}$ If the ghosts interact with the matter only via gravity, the decay rate per space-time volume is typically estimated to be of the order $\Gamma \sim \frac{\Lambda^{8}}{M_{\mathrm{Pl}}^{4}}$. This is to be contrasted to the measured flux of $\mathrm{MeV}$-photons [77], what yields the constraint on the cutoff scale $\Lambda \lesssim 3 \mathrm{MeV}$ [12]. The upper limit here is applied, provided that the sound speed is of the order unity, i.e., there is no hierarchy between the frequency and spatial momenta cutoffs. If $c_{s}^{2} \ll 1$, then the constraint on the spatial momenta cutoff can be relaxed by several orders of magnitude. The reason is twofold. First, there are

\footnotetext{
${ }^{11}$ This assumes that gravity remains unmodified at all the scales. Given that standard gravity must be embedded into some microscopic theory at the scales $\gtrsim M_{\mathrm{Pl}}$, a more conservative limit on the decay rate is $\Gamma \lesssim M_{\mathrm{Pl}}^{4}$. This is nevertheless many orders of magnitude larger than the phenomenologically allowed value.

${ }^{12}$ On the other hand, in the Lorentz-invariant theories, the cutoff is imposed on the center-of-mass energy $\sqrt{s}$ of the colliding particles. In this situation, the region of the integration over the spatial momenta is infinite. Namely, one can always boost the momenta, while keeping the quantity $\sqrt{s}$ unchanged.
} 
kinematic considerations, which severely constrain the phase space of the decay products for $c_{s}^{2} \ll 1$. Second, the Standard Model particles are coupled to the gravitational potential $\Psi$ (directly or via the graviton), which is different from the canonically normalized variable $\hat{\Psi}$ by a huge factor, once again depending on the sound speed. As a result, the effective coupling between the matter particles and the field $\hat{\Psi}$ is very small. With all the factors taken together, the decay rate turns out to be suppressed by a large power of the sound speed (compared to the case with the unit sound speed). In turn, this allows to extend the perturbative regime in the model to the $\mathrm{TeV}$ scales.

The discussion above is applied to the case where there is no direct interaction of the khronon field with the Standard Model particles. We specialize to this case in what follows. That is, we assume that the khronon affects the matter properties only via the mixing with the scalar gravitational potential $\Psi$. Apart from the vacuum stability issues, by the direct coupling of the khronon to the matter, one risks to reprocess the Lorentz-violating effects to the particle sector $[68,69] .{ }^{13}$ Typically, this is expected to modify the dispersion relation of the particles leading to a potential conflict with observational data [70-73].

We will be primarily interested in the processes with photons in the final state. These are argued to be the most relevant ones in ref. [12]. The action for electromagnetism is given by

$$
S_{\mathrm{el}}=-\frac{1}{4} \int d^{4} x \sqrt{-g} \cdot g^{\lambda \mu} g^{\rho \nu} F_{\mu \nu} F_{\lambda \rho},
$$

where $F_{\mu \nu} \equiv \nabla_{\nu} A_{\mu}-\nabla_{\mu} A_{\nu}=\partial_{\nu} A_{\mu}-\partial_{\mu} A_{\nu}$ is the electromagnetic tensor. The interactions of the photons with the ghosts are of two sorts: those following from the direct coupling of the photons to the scalar potential $\Psi$, and those involving an exchange of a graviton. In terms of the canonically normalized field $\hat{\Psi}$, the former are described by, ${ }^{14}$

$$
\mathcal{L}_{\mathrm{ph}-\mathrm{gh}} \sim \frac{\sqrt{\gamma}}{M_{\mathrm{Pl}}^{2}} \cdot \hat{\Psi} \cdot F_{\mu \nu}^{2}, \quad \frac{\gamma}{M_{\mathrm{Pl}}^{4}} \cdot \hat{\Psi}^{2} \cdot F_{\mu \nu}^{2} .
$$

Recall that the field $\hat{\Psi}$ is related to the scalar potential $\Psi$ by

$$
\hat{\Psi} \sim \frac{M_{\mathrm{Pl}}^{2}}{\sqrt{\gamma}} \Psi .
$$

At the tree level, the first interaction term on the r.h.s. of (3.1) leads to the process with two photons and one ghost particle in the final state. Naively, this should be the dominant one. In fact, it does not occur for the simple kinematic considerations discussed in subsection 3.1. The second interaction term on the r.h.s. of eq. (3.1) contributes to the process with two $\hat{\Psi}$-particles in the final state (see the left plot in figure 1), and is kinematically allowed. We estimate the associated vacuum decay rate in subsection 3.1. In particular, we will see that it gives a negligible contribution to the total decay rate compared to the interaction involving tensor degrees of freedom.

\footnotetext{
${ }^{13}$ In fact, Lorentz violation percolates the particle sector even in the absence of the direct coupling of the khronon to the standard matter, i.e., via quantum gravity loops [67]. However, this mechanism leads to the effects suppressed by the ratio of the UV cutoff $M_{*}$ and the Plank mass $M_{\mathrm{Pl}}$.

${ }^{14}$ One can write the analogous interactions but with the potential $\Phi$ instead of $\Psi$. These, however, give nothing new, because of the relation $\Psi=\Phi$, which holds in linear theory.
} 

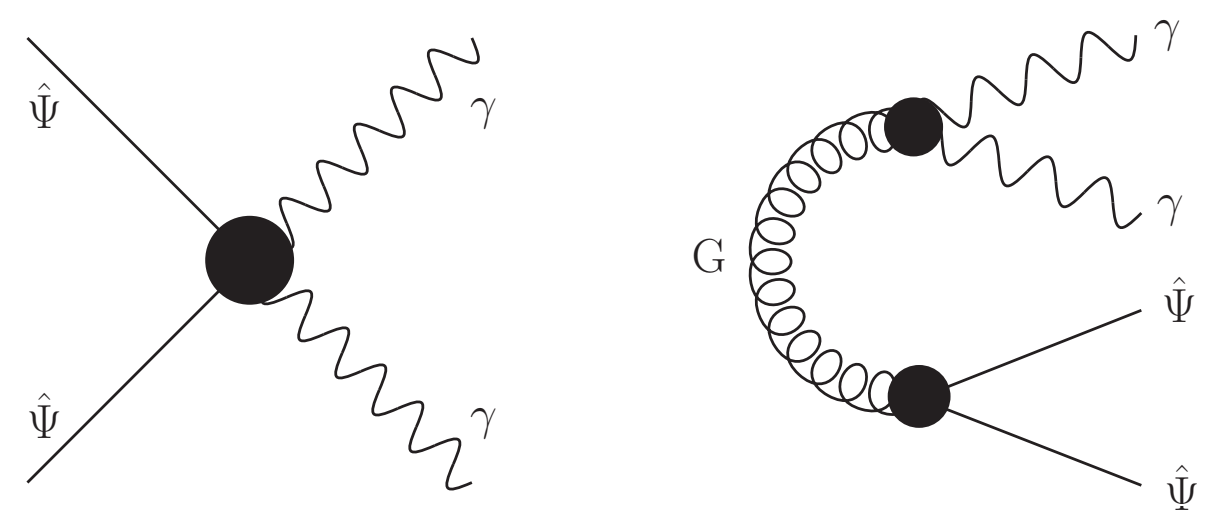

Figure 1. Two diagrams of the vacuum decay into a pair of photons (the wiggly lines labelled by ' $\gamma$ ') and a pair of ghosts (the straight lines labelled by ' $\hat{\Psi}^{\prime}$ '). The diagram on the left follows from the 4-point contact interaction (3.1). It gives the negligible contribution to the total vacuum decay rate. The leading contribution comes from the diagram on the right involving the propagator of the graviton (the springy line labelled by ' $\mathrm{G}$ ').

Schematically, the interaction of the photons with the tensor modes is given by the Lagrangian,

$$
\mathcal{L}_{T-p h} \sim \frac{1}{M_{\mathrm{Pl}}} \cdot \hat{h}^{\mu \nu} T_{\mu \nu}^{\mathrm{el}} \sim \frac{1}{M_{\mathrm{Pl}}} \hat{h}^{\mu \nu} F_{\mu \alpha} F^{\alpha}{ }_{\nu}
$$

Here $T_{\mu \nu}^{\mathrm{el}}$ is the electromagnetic stress-energy tensor; $\hat{h}^{\mu \nu}$ is the canonically normalized field of the helicity- 2 graviton related to the traceless part of the metric, $h^{\mu \nu}$, by

$$
\hat{h}^{\mu \nu} \sim M_{\mathrm{Pl}} \cdot h^{\mu \nu}
$$

Scalar-tensor interactions in the gravity sector are of the form,

$$
\mathcal{L}_{T-g h} \sim \frac{1}{M_{\mathrm{Pl}}} \hat{h}^{\mu \nu} T_{\mu \nu}^{\hat{\Psi}} \sim \frac{1}{M_{\mathrm{Pl}}}\left(\hat{h}^{00} \dot{\hat{\Psi}}^{2}+c_{s}^{2} \hat{h}^{i j} \partial_{i} \hat{\Psi} \partial_{j} \hat{\Psi}\right)
$$

where $T_{\mu \nu}^{\hat{\Psi}}$ is the stress-energy tensor of the scalar $\hat{\Psi}$. Note that the two terms inside the parentheses of eq. (3.4) are of the same order. Thus, we can estimate the strength of the scalar-tensor interaction simply by

$$
\mathcal{L}_{T-g h} \sim \frac{\gamma}{M_{\mathrm{Pl}}^{3}} \cdot \hat{h}^{i j} \partial_{i} \hat{\Psi} \partial_{j} \hat{\Psi} .
$$

The corresponding diagram of the vacuum decay into a couple of photons and ghosts is pictured in figure 1 (right plot), and contains the propagator of the graviton. We estimate the associated decay rate in subsection 3.2.

One comment is in order before we proceed. While we mainly focus on the electromagnetic interactions of the ghosts, there are a few more, which may trigger potentially dangerous processes. The first ones involve two gravitons/neutrinos in the final state. The corresponding interaction Lagrangians have the same order of magnitude as in the case of photons. However, those processes leave much weaker signatures in the observational 
data, and therefore, are practically undetectable. The process with an electron-positron pair in the final state is irrelevant for another reason. The pair carries the minimal energy $\sim 1 \mathrm{MeV}$, which must be balanced by the outgoing ghosts. We will see, however, that the energies of the particles produced in the vacuum decay do not exceed $\sim 1 \mathrm{keV}$. Hence, this process is forbidden.

\subsection{Vacuum decay into photons from the 4-point contact interaction with ghosts}

In view of our objectives, it will be enough to perform a rough estimation of the vacuum decay rate. In particular, we will ignore the interference between two diagrams in figure 1, and calculate the associated rates separately. We start with the case of the 4-point contact interaction between photons and ghosts (3.1). The corresponding matrix element is estimated by

$$
\mathcal{M}\left(\mathbf{k}_{1}, \mathbf{k}_{2} ; \mathbf{p}_{1}, \mathbf{p}_{2}\right) \sim \frac{\gamma}{M_{\mathrm{Pl}}^{4}} \cdot\left|\mathbf{k}_{1}\right| \cdot\left|\mathbf{k}_{2}\right| .
$$

We use the notation $\mathbf{k}_{i}$ for the momenta of the photons, and $\mathbf{p}_{i}$ for the momenta of the ghosts. Recall that the coefficient $\frac{\gamma}{M_{\mathrm{Pl}}^{4}}$ originates from the definition (3.2) of the canonically normalized variable $\hat{\Psi}$; the factor $\left|\mathbf{k}_{1}\right| \cdot\left|\mathbf{k}_{2}\right|$ stems from the derivative structure of the interactions in eq. (3.1). Modulo the irrelevant phase factors, the decay rate per spacetime volume is given by,

$$
\Gamma \sim \int \frac{d \mathbf{p}_{1}}{\left|\omega_{1}\right|} \frac{d \mathbf{p}_{2}}{\left|\omega_{2}\right|} \frac{d \mathbf{k}_{1}}{E_{1}} \frac{d \mathbf{k}_{2}}{E_{2}}\left|\mathcal{M}\left(\mathbf{k}_{1}, \mathbf{k}_{2} ; \mathbf{p}_{1}, \mathbf{p}_{2}\right)\right|^{2} \delta^{(4)}\left(p_{1}+p_{2}+k_{1}+k_{2}\right),
$$

where $\delta^{(4)}(\ldots)$ is the delta function, which ensures the conservation of the energy and momentum; $\omega_{i} \equiv p_{i}^{0}<0$ and $E_{i} \equiv k_{i}^{0}$ denote the energies of the ghosts and photons, respectively. To handle the integral in eq. (3.6), it is convenient to introduce the intermediate integrals over fictitious momenta $P$ and $K$ [74],

$$
\int d^{4} P \delta^{(4)}\left(P-p_{1}-p_{2}\right)=1, \quad \int d^{4} K \delta^{(4)}\left(K-k_{1}-k_{2}\right)=1 .
$$

Then, the decay rate can be written as follows,

$$
\Gamma \sim \frac{\gamma^{2}}{M_{\mathrm{Pl}}^{8}} \int d^{4} P d^{4} K \delta^{4}(K+P) \mathcal{I}_{1}(P) \mathcal{I}_{2}(K) .
$$

In the case of the direct coupling of the field $\Psi$ to the matter, the integrals $\mathcal{I}_{1}(P)$ and $\mathcal{I}_{2}(K)$ are defined as,

$$
\mathcal{I}_{1}(P)=\int \frac{d \mathbf{p}_{1}}{\left|\omega_{1}\right|} \frac{d \mathbf{p}_{2}}{\left|\omega_{2}\right|} \delta^{(4)}\left(P-p_{1}-p_{2}\right),
$$

and

$$
\mathcal{I}_{2}(K)=\int \frac{d \mathbf{k}_{1}}{E_{1}} \frac{d \mathbf{k}_{2}}{E_{2}}\left|\mathbf{k}_{1}\right|^{2} \cdot\left|\mathbf{k}_{2}\right|^{2} \delta^{(4)}\left(K-k_{1}-k_{2}\right) .
$$

These integrals can be evaluated in a straightforward manner. The result for the integral $\mathcal{I}_{1}(P)$ reads

$$
\mathcal{I}_{1}(P) \sim \frac{M_{\mathrm{Pl}}^{3}}{\gamma^{3 / 2}}
$$


(note that it is independent of the momentum $P$ ). Partially such a huge value of the integral is explained by the presence of the factors $\left|\omega_{i}\right|^{-1}$ in eq. (3.9) (recall the dispersion relation $\left.\left|\omega_{i}\right|=c_{s}\left|\mathbf{p}_{i}\right| \sim \frac{\sqrt{\gamma}}{M_{\mathrm{Pl}}}\left|\mathbf{p}_{i}\right|\right)$. The estimate for the integral $\mathcal{I}_{2}(K)$ is given by,

$$
\mathcal{I}_{2}(K) \sim K_{0}^{4} .
$$

Substituting eqs. (3.11) and (3.12) into eq. (3.8) and performing the integration over the fictitious 4-momentum $P$, we get the following estimate for the decay rate,

$$
\Gamma \sim \frac{\sqrt{\gamma}}{M_{\mathrm{Pl}}^{5}} \int d^{4} K K_{0}^{4}
$$

In order to understand the region of integration over the momentum $K$, one should include kinematic considerations. So, one has

$$
|\mathbf{K}| \lesssim K_{0}=\left|\mathbf{k}_{1}\right|+\left|\mathbf{k}_{2}\right|=c_{s}\left(\left|\mathbf{p}_{1}\right|+\left|\mathbf{p}_{2}\right|\right) .
$$

Recall now that the spatial momenta of the ghost particles are bounded from above by the strong coupling scale, above which the theory is assumed to be free of instabilities. Hence, $|\mathbf{K}| \lesssim K_{0} \lesssim c_{s} \Lambda_{p}$. As it follows, for $c_{s}^{2} \ll 1$, the momenta of the photons are much smaller than those of the ghost particles. Consequently, for the momentum conservation equation to be obeyed, the outgoing ghost particles must be practically anti-collinear.

Using kinematic considerations, we obtain an order of magnitude expression for the decay rate,

$$
\Gamma \sim \frac{\gamma^{9 / 2} \Lambda_{p}^{8}}{M_{\mathrm{Pl}}^{13}}
$$

We see explicitly the huge suppression by a large power of the Planck mass. This fact becomes particularly prominent upon substituting the estimate for the strong coupling scale, i.e., $\Lambda_{p} \propto \frac{\gamma^{3 / 4}}{M_{\mathrm{Pl}}^{1 / 2}}$. We obtain,

$$
\Gamma \sim \frac{\gamma^{21 / 2}}{M_{\mathrm{Pl}}^{17}} .
$$

By contrasting this decay rate to the observed flux of photons on the Earth, one can extract a constraint on the parameter $\sqrt{\gamma}$ and, consequently, on the strong coupling scale $\Lambda_{p}$. As we will see in the next subsection, however, the decay rate (3.13) is sub-dominant compared to that of the process involving an exchange by the graviton. Hence, the resulting constraints are expected to be milder.

Here let us pause for an instant to show that the process with two photons and one ghost in the final state is indeed forbidden. This follows from kinematic considerations similar to those discussed above. The momentum $\mathbf{p}$ of the (only) ghost is defined from the conservation equation, i.e., $\mathbf{p}=-\mathbf{k}_{1}-\mathbf{k}_{2}$, where $\mathbf{k}_{1}$ and $\mathbf{k}_{2}$ are the momenta of the photons. The total energy of the particles in the final state is given by,

$$
\left|\mathbf{k}_{1}\right|+\left|\mathbf{k}_{2}\right|-c_{s}\left|\mathbf{k}_{1}+\mathbf{k}_{2}\right| \gtrsim\left(\left|\mathbf{k}_{1}\right|+\left|\mathbf{k}_{2}\right|\right) \cdot\left(1-c_{s}\right)>0 .
$$

The inequality on the r.h.s. implies that the energy conservation equation in the process with one ghost cannot be obeyed. Consequently, this process does not occur. It is straightforward to generalize the latter statement to any process of the vacuum decay with one ghost particle in the final state. 


\subsection{Vacuum decay into photons mediated by the exchange of a graviton}

The matrix element for the process involving an exchange by the graviton is estimated as,

$$
\mathcal{M}\left(\mathbf{k}_{1}, \mathbf{k}_{2} ; \mathbf{p}_{1}, \mathbf{p}_{2}\right) \sim \frac{\gamma}{M_{\mathrm{Pl}}^{4}} \frac{\left(\left|\mathbf{k}_{1}\right| \cdot\left|\mathbf{k}_{2}\right|\right) \cdot\left(\left|\mathbf{p}_{1}\right| \cdot\left|\mathbf{p}_{2}\right|\right)}{q^{2}} .
$$

Here $\left(q^{2}\right)^{-1}=\left[\left(\omega_{1}+\omega_{2}\right)^{2}-\left(\mathbf{p}_{1}+\mathbf{p}_{2}\right)^{2}\right]^{-1}$, - the propagator of the graviton. The decay rate is estimated by the same generic expression (3.6), now with the matrix element (3.14) substituted in. Again introducing the integration over the fictitious momenta $P$ and $K$, one can write the decay rate as in eq. (3.8) with the integral $\mathcal{I}_{2}(K)$ still given by eq. (3.10), and the integral $\mathcal{I}_{1}(P)$ defined by,

$$
\mathcal{I}_{1}(P)=\frac{1}{P^{4}} \int \frac{d \mathbf{p}_{1}}{\left|\omega_{1}\right|} \frac{d \mathbf{p}_{2}}{\left|\omega_{2}\right|} \cdot\left|\mathbf{p}_{1}\right|^{2} \cdot\left|\mathbf{p}_{2}\right|^{2} \delta^{(4)}\left(P-p_{1}-p_{2}\right) .
$$

The factor $1 / P^{4}$ stems from the propagator of the graviton. To evaluate this integral, it is convenient to make a redefinition of the variable $P_{0}=c_{s} \tilde{P}_{0}$. Then, the value of the integral can be estimated on the simple dimensional grounds,

$$
\mathcal{I}_{1}(P) \sim \frac{1}{P^{4}} \frac{M_{\mathrm{Pl}}^{3}}{\gamma^{3 / 2}} \tilde{P}_{0}^{4} \sim \frac{1}{P^{4}} \frac{M_{\mathrm{Pl}}^{7}}{\gamma^{7 / 2}} P_{0}^{4} \sim \frac{M_{\mathrm{Pl}}^{7}}{\gamma^{7 / 2}} .
$$

Note that compared to the case with the direct coupling to the field $\Psi$, we gained the amplification factor $M_{\mathrm{Pl}}^{4} / \gamma^{2}$. Therefore, the resulting decay rate is parametrically larger,

$$
\Gamma \sim \frac{\gamma^{17 / 2}}{M_{\mathrm{Pl}}^{13}}
$$

Now, let us contrast our theoretical prediction of the vacuum decay rate to the experimental data. The number density $n$ of the produced photons is related to the quantity $\Gamma$ by [12]

$$
n \simeq \Gamma t_{0},
$$

where $t_{0}$ denotes the age of the Universe, $t_{0} \sim H_{0}^{-1}$, and $H_{0}$ is the current Hubble rate. On the other hand, the measured flux $F$ of the photons in the range of energies $E_{\mathrm{ph}}$ corresponding to the X-rays $(\mathrm{keV})$ and gamma-ray bursts is estimated from [75-77]

$$
F \cdot E_{\mathrm{ph}} \sim A \cdot \frac{\mathrm{keV}}{\mathrm{s} \cdot \mathrm{cm}^{2} \cdot \mathrm{sr}} .
$$

We introduced the fictitious dimensionless parameter $A$, which ranges between 1 and 100 . The uncertainty here accounts for the slight energy dependence of the quantity $F \cdot E_{\mathrm{ph}}$. We demand that the flux of the photons originating from the vacuum decay does not exceed the observed one, and that yields the upper bound on the parameter $\sqrt{\gamma}$,

$$
\sqrt{\gamma} \lesssim 10^{9} \mathrm{GeV}
$$

(practically independent of the uncertainty on the parameter $A$ ). This sets the limit on the strong coupling scale in the theory,

$$
\Lambda_{p} \lesssim 10 \mathrm{TeV},
$$


(accidentally, it coincides with the scale of experiments at the LHC). The constraint (3.16) is seven orders of magnitude less stringent than the limit of ref. [12] deduced assuming the standard dispersion relation for the ghosts. Furthermore, eq. (3.16) demonstrates thirty orders of magnitude improvement compared to the constraint obtained in the branch of the model plagued by gradient instabilities.

Constraints (3.15) and (3.16) imply that the maximal energies of the produced particles lie in the keV-range. This follows from eq. (2.29),

$$
\Lambda_{\omega} \lesssim 1 \mathrm{keV}
$$

Therefore, the comparison with the flux of the cosmic X-rays is justified. The result (3.17) has immediate consequences for the series of processes would be going naively in the presence of the ghosts. First, it forbids the vacuum decay with an electron-positron pair in the final state. Indeed, the minimal mass of the pair is of the order $\mathrm{MeV}$ and is parametrically larger than that allowed by eq. (3.17). For the similar reasons, the constraint on the energy of the produced ghosts makes it impossible for lighter particles to decay into heavier ones [78]. So, the hypothetic processes of the electron decay into the muon and two neutrinos, i.e., $e^{-} \rightarrow \mu^{-}+\nu_{e}+\bar{\nu}_{\mu}+$ ghosts, or the proton decay with the neutron, positron and neutrino in the final state, i.e., $p \rightarrow n+e^{+}+\nu_{e}+$ ghosts, do not occur.

In the remainder of the section, we comment on the alternative ways to constrain the parameter $\sqrt{\gamma}$ and the strong coupling scale $\Lambda_{p}$. First, the vacuum decay triggered by the direct coupling of the ghosts to the photons is characterized by the smaller rate and, hence, results into milder limits on those parameters. These read $\sqrt{\gamma} \lesssim 10^{11} \mathrm{GeV}$ and $\Lambda_{p} \lesssim 10^{4} \mathrm{TeV}$.

Stronger constraints, of the order of those given in eqs. (3.15) and (3.16), follow from cosmological considerations. That is, one does not want to overproduce radiation. Indeed, the energy density of the photons originating from the vacuum decay is estimated by

$$
\rho_{\mathrm{ph}} \sim c_{s} \Lambda_{p} \Gamma t_{0} .
$$

The factor $c_{s} \Lambda_{p}$ stands for the maximal energy of the photons, while the factor $\Gamma t_{0}$ accounts for their number per unit volume. This should not exceed the total energy density of radiation in the Universe, $\rho_{\text {rad }} \sim 10^{-5} H_{0}^{2} M_{\mathrm{Pl}}^{2}$. Substituting the numbers, we again obtain the upper limit (3.16) on the strong coupling scale.

Note that the cosmological constraint taken separately could be essentially relaxed for the following reasons. The energy of the ghosts produced in the vacuum decay is equal to that of the photons, but has opposite sign. Furthermore, the condensate of the ghosts is characterized by a radiation-like equation of state. Hence, at least naively, the vacuum decay with the photons in the final state does not affect the cosmological evolution appreciably. This potentially interesting loophole is irrelevant for our discussion, - the constraint (3.16) obtained from the direct observation of keV-photons is strong enough to not worry about any consequences for cosmology.

The constraint (3.15) on the parameter $\sqrt{\gamma}$ may have some applications for the Dark Matter physics (albeit, perhaps, futuristic). Converting it to the upper bound on the sound 
speed squared, we have, ${ }^{15}$

$$
c_{s}^{2} \lesssim 10^{-20}
$$

This is 10 orders of magnitude stronger than the limit deduced from the galaxy formation considerations. Namely, for the bottom-up picture of the large scale structure formation to occur, the constraint $c_{s}^{2} \lesssim 10^{-10}$ must be applied [38]. The upper bound here corresponds to the situation, when the formation of dwarf galaxies is suppressed. Such a large value of the sound speed squared could be relevant for the so called missing satellite problem [40, 79, 80], - the observed number of the dwarf galaxies is much smaller than the one predicted in the Cold Dark Matter framework. Note, however, that the mismatch between the observations and the theoretical expectation can be relatively simply explained by the effects of the baryonic physics [81], - hence, it is not an immediate source of worry.

\section{Discussion}

In the present paper, we showed that the strong coupling scale of the projectable HořavaLifshitz gravity can be raised to $10 \mathrm{TeV}$, upon switching to the ghost unstable branch of the scenario. This is certainly an advantage over the branch plagued by the gradient instabilities, since now we do not have the problems with recovering GR at large distances. We reiterate, however, that the presence of the strong coupling by itself (even a high one) leads to the loss of perturbativity in the Hořava-Lifshitz gravity. In this sense, prospects for renormalizing the model, - the original motivation behind the Hořava-Lifshitz gravity, - still remain unclear.

Meanwhile, keeping in mind the problems with the UV completion, we can enjoy the rich phenomenology of the model. Perhaps, the most relevant one is the Dark Matter. This is quite a generic prediction of the IR modifications of gravity involving the spontaneous breaking of the Lorentz symmetry, e.g., the ghost condensate $[14,16]$. Not surprisingly, the same observation has been made also in the context of the projectable Hořava-Lifshitz gravity [27, 28]. The specific feature of this Dark Matter is that the fluid elements always follow geodesics equation, as it is indicated by the constraint (2.9). This typically implies a pathology in the model, - attracted by the gravitational force, trajectories corresponding to the different fluid elements cross at finite times leading to the so-called caustic singularities. ${ }^{16}$ On the other hand, that conclusion may alter due to the presence of the higher derivative term as in eq. (2.10), which may smoother the caustic singularity. The corresponding mechanism has been discussed in refs. [28, 38] and stems from the possibility to have regions in space, where gravity acts as a repulsive force (namely, it turns into antigravity). However, numerical simulations capable of verifying or ruling out this mechanism are still pending.

Particle production caused by the vacuum decay opens up an intriguing opportunity to reheat the Universe even without inflation. Note that for this scenario to be realized, the parameter $\gamma$ must be sufficiently large at very early times. Otherwise, one would be

\footnotetext{
${ }^{15}$ The lower limit on the sound speed can be inferred from eq. (2.31). It reads, $c_{s}^{2} \gtrsim 10^{-42}$.

${ }^{16}$ See refs. $[82,83]$ for the examples of caustic singularities in the models with the non-canonical scalar fields.
} 
able to produce only very low energetic particles. In fact, the constant $\gamma$ is implied to follow the renormalization group flow starting from the values of the order of the Planck mass squared. Apart from the obscure quantum gravity issues, the time dependence of $\gamma$ appears to be necessary for the production of the Dark Matter with the correct initial conditions $[39,84]$.

From somewhat more down-to-earth perspective, the production of the keV-photons out of the vacuum may strongly affect the equilibrium in the early Universe. In particular, the injection of the out-of-equilibrium photons at the redshifts $z \lesssim 10^{6}$ is expected to strongly modify the black-body spectrum of the Cosmic Microwave Background [85]. ${ }^{17}$ It would be certainly interesting to contrast the values of the $\mu$ and $y$-type distortions following from the scenario at hand with the associated COBE/FIRAS limits, and furthermore make the predictions for the future PIXIE data. This might be one promising way to improve the constraint on the parameter $\gamma$. At even higher redshifts, the presence of the keVphotons may have some impact on the Big Bang nucleosynthesis. Note, however, that the bound energies of the nuclei are much larger and, thus, do not get destroyed by the injection of the soft photons. Nevertheless, keV-photons may change the conditions at which nucleosynthesis proceeds.

\section{Acknowledgments}

We are indebted to E. Babichev, N. Bartolo, D. Blas, M. Ivanov, P. Karmakar, M. Pshirkov and A. Vikman for many useful comments and discussions. The work of FA is supported by the National Taiwan University (NTU) under Project No. 103R4000 and by the NTU Leung Center for Cosmology and Particle Astrophysics (LeCosPA) under Project No. FI121.

\section{A On the equivalence between the IR limit of projectable Hořava-Lifshitz gravity and mimetic matter scenario}

At the end of subsection 2.1, we pointed out that the action for the IR limit of the projectable Hořava-Lifshitz gravity can be written in the form (2.10). On the other hand, eq. (2.10) describes the dynamics of the version of the mimetic matter scenario considered in refs. [37-39, 51]. Hence, results of the present paper can be literally translated into the latter context. The fact that the projectable Hořava-Lifshitz gravity and the mimetic matter scenario are equivalent was first pointed out in ref. [43]. ${ }^{18}$ The present appendix serves to prove this statement rigorously.

\footnotetext{
${ }^{17}$ Although, $\mathrm{keV}$ photons are quite separated from the CMB photons by a large energy gap, things were different at redshifts $z \lesssim 10^{6}$, where the CMB distortions are expected to be produced. Namely, in that case, the energy of $\mathrm{CMB}$ photons was in the sub-keV range, thus making them particularly vulnerable to the energy of non-equilibrium photons.

${ }^{18}$ More precisely, ref. [43] links the projectable Hořava-Lifshitz gravity to the version of the scalar EinsteinAether model considered in ref. [44]. However, comparing refs. [37-39, 51] with ref. [44], one recognizes the latter as a generalization of the mimetic matter scenario.
} 
The proof of the equivalence is based on the following observation. Let us consider the action of the form,

$$
S=\int \mathcal{L}(\chi, f)+\lambda\left(f-F\left(\chi, \partial_{\mu} \chi, \partial_{\mu \nu} \chi, \ldots\right)\right) .
$$

Here $\mathcal{L}(\chi, f)$ is the Lagrange function of some variables $\chi$ and $f$ as well as their derivatives, and the Lagrange multiplier $\lambda$ enforces the constraint $f=F\left(\chi, \partial_{\mu} \chi, \partial_{\mu \nu} \chi, \ldots\right)$, so that the field $f$ is a function $F$ of the field $\chi$ and its derivatives. Then, one can show that the dynamics of the field $\chi$ generated by eq. (A.1) is equivalent to that following from the reduced action,

$$
S_{r}=\left.\int \mathcal{L}(\chi, f)\right|_{f=F\left(\chi, \partial_{\mu} \chi, \partial_{\mu \nu} \chi, \ldots\right)} .
$$

The proof of this statement is given in the Comment 1 of section 2 of ref. [63] (see also the references therein), and we do not repeat it here. We would like to emphasize that the equivalence between the actions (A.1) and (A.2) is not exact, but only with respect to the dynamics generated for the field $\chi$. Indeed, variation of the action (A.1) with respect to the field $f$ gives rise to the equation of motion, which is absent in the case of the action (A.2). This observation will be important for our further discussion.

Now, let us consider the 'true' action for the projectable Hořava-Lifshitz gravity, i.e., before substituting the constraint $g^{\mu \nu} \partial_{\mu} \varphi \partial_{\nu} \varphi=1$. Combining eqs. (2.6), (2.7) and (2.8) we get,

$$
S_{\mathrm{IR}}=S_{\mathrm{GR}}+\int d^{4} x \sqrt{-g}\left[\frac{\tilde{\Sigma}}{2}\left(g^{\mu \nu} \partial_{\mu} \varphi \partial_{\nu} \varphi-1\right)+\frac{\gamma}{2}\left(\nabla_{\mu}\left(\frac{\nabla^{\mu} \varphi}{\sqrt{g^{\mu \nu} \partial_{\mu} \varphi \partial_{\nu} \varphi}}\right)\right)^{2}\right] .
$$

Here we introduced the notation $\tilde{\Sigma}$ for the Lagrange multiplier field to avoid the confusion in the future. Below we also repeat the action (2.10) of the mimetic matter scenario for the convenience of the references,

$$
S_{\mathrm{mim}}=S_{\mathrm{GR}}+\int d^{4} x \sqrt{-g}\left[\frac{\Sigma}{2}\left(g^{\mu \nu} \partial_{\mu} \varphi \partial_{\nu} \varphi-1\right)+\frac{\gamma}{2}(\square \varphi)^{2}\right] .
$$

The point is to show that both actions (A.3) and (A.4) are equivalent to the following one,

$$
S=S_{\mathrm{GR}}+\int d^{4} x \sqrt{-g}\left[\frac{\Sigma}{2}\left(g^{\mu \nu} \partial_{\mu} \varphi \partial_{\nu} \varphi-X\right)+\frac{\tilde{\Sigma}}{2}(X-1)+\frac{\gamma}{2}\left(\nabla_{\mu}\left(\frac{\nabla^{\mu} \varphi}{\sqrt{X}}\right)\right)^{2}\right],
$$

and, therefore, they are equivalent between each other. In eq. (A.5), $X$ is the new variable, which obeys the constraint $X=1$ enforced by the Lagrange multiplier field $\tilde{\Sigma}$. First, it is obvious that the actions (A.5) and (A.4) match the generic ones (A.1) and (A.2), respectively, with the field $\lambda$ in eq. (A.1) understood as the Lagrange multiplier $\tilde{\Sigma}$ in eq. (A.5). Hence, eqs. (A.5) and (A.4) describe the same dynamics with respect to the fields $\Sigma, \varphi$ and the metric $g^{\mu \nu}$. On the other hand, the actions (A.5) and (A.3) are analogous to those of eqs. (A.1) and (A.2), respectively, with the field $\lambda$ in eq. (A.1) understood as the Lagrange multiplier $\Sigma$ in eq. (A.5), and the associated constraint given by $X=g^{\mu \nu} \partial_{\mu} \varphi \partial_{\nu} \varphi$. 
Hence, the actions (A.5) and (A.4) describe the same dynamics with respect to the fields $\tilde{\Sigma}$, $\varphi$ and the metric $g^{\mu \nu}$. To summarize, the actions eqs. (A.3), (A.4), (A.5), and, consequently, the IR limit of the projectable Hořava-Lifshitz gravity and the mimetic matter scenario, are equivalent.

Let us make one important observation here. Strictly speaking, the discussion above implies only the equivalence in the dynamics of the metric $g^{\mu \nu}$ as well as the khronon field $\varphi$. Namely, provided that they start from the same initial conditions in both models, they follow the same evolution at later times. However, this is not true for the fields $\Sigma$ and $\tilde{\Sigma}$ (that is why we chose the different notations for them). ${ }^{19}$ To understand the difference in treating the Lagrange multiplier fields in two models, it is enough to vary the action (A.5) with respect to the field $X$, and then set the latter to unity in the end. This gives,

$$
\Sigma-\tilde{\Sigma}=\gamma \nabla_{\rho} \square \varphi \nabla^{\rho} \varphi
$$

Therefore, one should be cautious, when keeping the fields $\Sigma$ and $\tilde{\Sigma}$ as the independent variables. In particular, setting them to be equal at the initial Cauchy surface will result into different dynamics in the two models. On the other hand, with the choice of the independent variables $\left(\varphi, g^{\mu \nu}\right),{ }^{20}$ the dynamics will be the same, i.e., the predictions of the two models will be physically indistinguishable. The technical reason is that the shift (A.6) is exactly compensated by the associated shift of the stress-energy tensor calculated in the IR limit of the projectable Hořava-Lifshitz gravity $T_{\mu \nu}^{\mathrm{IR}}$ compared to that of the mimetic matter scenario $T_{\mu \nu}^{\mathrm{mim}}$. Namely, these are related to each other by,

$$
T_{\mu \nu}^{\mathrm{IR}}=T_{\mu \nu}^{\operatorname{mim}}(\Sigma \rightarrow \tilde{\Sigma})+\gamma \nabla_{\rho} \varphi \nabla^{\rho} \square \varphi \nabla_{\mu} \varphi \nabla_{\nu} \varphi
$$

where the tensor $T_{\mu \nu}^{\operatorname{mim}}$ is given by [37],

$$
T_{\mu \nu}^{\operatorname{mim}}=\Sigma \nabla_{\mu} \varphi \nabla_{\nu} \varphi+\gamma\left(\nabla_{\mu} \varphi \nabla_{\mu} \square \varphi+\frac{1}{2}(\square \varphi)^{2}\right) g_{\mu \nu}-\gamma\left(\nabla_{\nu} \varphi \nabla_{\mu} \square \varphi+\nabla_{\nu} \square \varphi \nabla_{\mu} \varphi\right) .
$$

Combining eqs. (A.6), (A.7) and (A.8), we get $T_{\mu \nu}^{\mathrm{IR}}-T_{\mu \nu}^{\operatorname{mim}}=\left(\tilde{\Sigma}-\Sigma+\gamma \nabla_{\rho} \varphi \nabla^{\rho} \square \varphi\right) \nabla_{\mu} \varphi \nabla_{\nu} \varphi=$ 0 . This completes the proof.

\section{B Curing ghost instabilities beyond projectable Hořava-Lifshitz gravity}

Here, we briefly discuss one possible way to simultaneously cure ghost instabilities and retain the model perturbative. For this purpose, we include the operator (2.23) into the analysis. That operator, we remind, has a dimension higher than marginal. Therefore, it was not considered in the bulk of the paper. In the unitary gauge, the quadratic action for

\footnotetext{
${ }^{19}$ As the attentive reader could notice, the reason is that the projectable Hor̆ava-Lifshitz model is equivalent to the model (A.5) with respect to the set of the fields $(\tilde{\Sigma}, \varphi, \Psi, \ldots)$. At the same time, the mimetic matter scenario is equivalent to the model (A.5) with respect to the set of the fields $(\Sigma, \varphi, \Psi, \ldots)$.

${ }^{20}$ This has been indeed our strategy in subsection 2.2. That is, the Lagrange multiplier field is not present in the quadratic action (2.15): it drops off upon substituting the constraint (2.14).
} 
an extended model including the UV operators (2.21) is given by

$$
\begin{aligned}
\delta_{2} S= & \frac{1}{16 \pi G} \int d^{4} x\left[-6 \dot{\Psi}^{2}-2 \Psi \Delta \Psi+4 \dot{\Psi}(\Delta \dot{E}-\Delta B)+\frac{\alpha}{M_{*}^{2}} \Psi \Delta^{2} \Psi+\ldots\right]+ \\
& +\frac{\gamma}{2} \int d^{4} x \cdot[3 \dot{\Psi}+\Delta B-\Delta \dot{E}] \hat{A}[3 \dot{\Psi}+\Delta B-\Delta \dot{E}]
\end{aligned}
$$

where we assume the Minkowski background. Here $\alpha$ is an order one constant governing the relevant operator and the ellipsis stand for the contributions from the marginal operators. We introduced the operator $\hat{A}$ defined by,

$$
\hat{A} \equiv\left(1-\frac{\beta M_{\mathrm{Pl}}^{2}}{\gamma M_{*}^{2}} \Delta\right)
$$

where $\beta$ is an order one constant governing the operator (2.23).

Varying the action (B.1) with respect to the combination $B-\dot{E}$, one gets the constraint equation,

$$
\Delta B-\Delta \dot{E}=\frac{\dot{\Psi}}{4 \pi \gamma G \hat{A}}-3 \dot{\Psi} .
$$

Substituting this back into the action (B.1), one gets

$$
\delta_{2} S=\int d^{4} x\left(6 \dot{\Psi}^{2}-\frac{\dot{\Psi}^{2}}{2 \pi \gamma G \hat{A}}-2 \Psi \Delta \Psi+\frac{\alpha}{M_{*}^{2}} \Psi \Delta^{2} \Psi\right) .
$$

Switching to the Fourier space analysis, we observe that for small momenta $|\mathbf{p}| \lesssim M_{*}$, the second term on the r.h.s. dominates over the first one. Hence, the kinetic term has a ghost-like sign in this regime. On the other hand, for larger momenta $|\mathbf{p}| \gtrsim M_{*}$, the first term is dominant, and we recover the positive sign of the kinetic term.

This solution is not without problems, though. Indeed, that way of curing ghost instabilities is at risk of getting gradient instabilities instead. The problem can be avoided by a proper tuning of the constants $\alpha$ and $\beta$.

Open Access. This article is distributed under the terms of the Creative Commons Attribution License (CC-BY 4.0), which permits any use, distribution and reproduction in any medium, provided the original author(s) and source are credited.

\section{References}

[1] Planck collaboration, P.A.R. Ade et al., Planck 2015 results. XIII. Cosmological parameters, arXiv:1502.01589 [INSPIRE].

[2] M. Fierz and W. Pauli, On relativistic wave equations for particles of arbitrary spin in an electromagnetic field, Proc. Roy. Soc. Lond. A 173 (1939) 211 [InSPIRE].

[3] D.G. Boulware and S. Deser, Can gravitation have a finite range?, Phys. Rev. D 6 (1972) 3368 [INSPIRE].

[4] A.I. Vainshtein, To the problem of nonvanishing gravitation mass, Phys. Lett. B 39 (1972) 393 [INSPIRE]. 
[5] K. Hinterbichler, Theoretical Aspects of Massive Gravity, Rev. Mod. Phys. 84 (2012) 671 [arXiv:1105.3735] [INSPIRE].

[6] C. de Rham, Massive Gravity, Living Rev. Rel. 17 (2014) 7 [arXiv:1401.4173] [InSPIRE].

[7] V.A. Rubakov and P.G. Tinyakov, Infrared-modified gravities and massive gravitons, Phys. Usp. 51 (2008) 759 [arXiv:0802.4379] [INSPIRE].

[8] D. Comelli, F. Nesti and L. Pilo, Nonderivative Modified Gravity: a Classification, JCAP 11 (2014) 018 [arXiv: 1407.4991] [INSPIRE].

[9] D. Comelli, F. Nesti and L. Pilo, Massive gravity: a General Analysis, JHEP 07 (2013) 161 [arXiv: 1305.0236] [INSPIRE].

[10] D. Comelli, F. Nesti and L. Pilo, Cosmology in General Massive Gravity Theories, JCAP 05 (2014) 036 [arXiv: 1307.8329] [INSPIRE].

[11] V.A. Rubakov, Lorentz-violating graviton masses: Getting around ghosts, low strong coupling scale and VDVZ discontinuity, hep-th/0407104 [INSPIRE].

[12] J.M. Cline, S. Jeon and G.D. Moore, The Phantom menaced: Constraints on low-energy effective ghosts, Phys. Rev. D 70 (2004) 043543 [hep-ph/0311312] [INSPIRE].

[13] S.L. Dubovsky, Phases of massive gravity, JHEP 10 (2004) 076 [hep-th/0409124] [INSPIRE].

[14] N. Arkani-Hamed, H.-C. Cheng, M.A. Luty and S. Mukohyama, Ghost condensation and a consistent infrared modification of gravity, JHEP 05 (2004) 074 [hep-th/0312099] [INSPIRE].

[15] N. Arkani-Hamed, P. Creminelli, S. Mukohyama and M. Zaldarriaga, Ghost inflation, JCAP 04 (2004) 001 [hep-th/0312100] [INSPIRE].

[16] N. Arkani-Hamed, H.-C. Cheng, M.A. Luty, S. Mukohyama and T. Wiseman, Dynamics of gravity in a Higgs phase, JHEP 01 (2007) 036 [hep-ph/0507120] [INSPIRE].

[17] P. Hořava, Quantum Gravity at a Lifshitz Point, Phys. Rev. D 79 (2009) 084008 [arXiv: 0901.3775] [INSPIRE].

[18] P. Hořava, Membranes at Quantum Criticality, JHEP 03 (2009) 020 [arXiv:0812.4287] [INSPIRE].

[19] P. Hořava, Spectral Dimension of the Universe in Quantum Gravity at a Lifshitz Point, Phys. Rev. Lett. 102 (2009) 161301 [arXiv:0902.3657] [INSPIRE].

[20] C. Charmousis, G. Niz, A. Padilla and P.M. Saffin, Strong coupling in Hořava gravity, JHEP 08 (2009) 070 [arXiv: 0905.2579] [inSPIRE].

[21] D. Blas, O. Pujolàs and S. Sibiryakov, On the Extra Mode and Inconsistency of Hor̆ava Gravity, JHEP 10 (2009) 029 [arXiv:0906.3046] [INSPIRE].

[22] D. Blas, O. Pujolàs and S. Sibiryakov, Consistent Extension of Hor̆ava Gravity, Phys. Rev. Lett. 104 (2010) 181302 [arXiv: 0909.3525] [INSPIRE].

[23] D. Blas, O. Pujolàs and S. Sibiryakov, Models of non-relativistic quantum gravity: The Good, the bad and the healthy, JHEP 04 (2011) 018 [arXiv: 1007.3503] [INSPIRE].

[24] C. Germani, A. Kehagias and K. Sfetsos, Relativistic Quantum Gravity at a Lifshitz Point, JHEP 09 (2009) 060 [arXiv:0906.1201] [inSPIRE].

[25] K. Koyama and F. Arroja, Pathological behaviour of the scalar graviton in Horava-Lifshitz gravity, JHEP 03 (2010) 061 [arXiv:0910.1998] [INSPIRE]. 
[26] A.O. Barvinsky, D. Blas, M. Herrero-Valea, S.M. Sibiryakov and C.F. Steinwachs, Renormalization of Hořava gravity, Phys. Rev. D 93 (2016) 064022 [arXiv:1512.02250] [INSPIRE].

[27] S. Mukohyama, Dark matter as integration constant in Hor̆ava-Lifshitz gravity, Phys. Rev. D 80 (2009) 064005 [arXiv: 0905.3563] [INSPIRE].

[28] S. Mukohyama, K. Nakayama, F. Takahashi and S. Yokoyama, Phenomenological Aspects of Hořava-Lifshitz Cosmology, Phys. Lett. B 679 (2009) 6 [arXiv:0905. 0055] [InSPIRE].

[29] S. Mukohyama, Hořava-Lifshitz Cosmology: A Review, Class. Quant. Grav. 27 (2010) 223101 [arXiv: 1007.5199 ] [INSPIRE].

[30] S. Mukohyama, Scale-invariant cosmological perturbations from Hořava-Lifshitz gravity without inflation, JCAP 06 (2009) 001 [arXiv:0904.2190] [INSPIRE].

[31] S. Mukohyama, Caustic avoidance in Hořava-Lifshitz gravity, JCAP 09 (2009) 005 [arXiv: 0906.5069] [INSPIRE].

[32] J. Garriga and A. Vilenkin, Living with ghosts in Lorentz invariant theories, JCAP 01 (2013) 036 [arXiv: 1202.1239] [INSPIRE].

[33] D.E. Kaplan and R. Sundrum, A Symmetry for the cosmological constant, JHEP 07 (2006) 042 [hep-th/0505265] [INSPIRE].

[34] S. Dyda, E.E. Flanagan and M. Kamionkowski, Vacuum Instability in Chern-Simons Gravity, Phys. Rev. D 86 (2012) 124031 [arXiv:1208.4871] [INSPIRE].

[35] D. Blas, O. Pujolàs and S. Sibiryakov, Comment on 'Strong coupling in extended Hořava-Lifshitz gravity', Phys. Lett. B 688 (2010) 350 [arXiv:0912.0550] [INSPIRE].

[36] A.H. Chamseddine and V. Mukhanov, Mimetic Dark Matter, JHEP 11 (2013) 135 [arXiv:1308.5410] [INSPIRE].

[37] A.H. Chamseddine, V. Mukhanov and A. Vikman, Cosmology with Mimetic Matter, JCAP 06 (2014) 017 [arXiv: 1403.3961] [INSPIRE].

[38] F. Capela and S. Ramazanov, Modified Dust and the Small Scale Crisis in CDM, JCAP 04 (2015) 051 [arXiv: 1412.2051] [INSPIRE].

[39] L. Mirzagholi and A. Vikman, Imperfect Dark Matter, JCAP 06 (2015) 028 [arXiv: 1412.7136] [INSPIRE].

[40] D.H. Weinberg, J.S. Bullock, F. Governato, R.K. de Naray and A.H.G. Peter, Cold dark matter: controversies on small scales, arXiv:1306.0913 [INSPIRE].

[41] N. Deruelle and J. Rua, Disformal Transformations, Veiled General Relativity and Mimetic Gravity, JCAP 09 (2014) 002 [arXiv: 1407.0825] [INSPIRE].

[42] T. Jacobson and D. Mattingly, Gravity with a dynamical preferred frame, Phys. Rev. D 64 (2001) 024028 [gr-qc/0007031] [INSPIRE].

[43] T. Jacobson and A.J. Speranza, Comment on "Scalar Einstein-Aether theory", arXiv:1405.6351 [INSPIRE].

[44] Z. Haghani, T. Harko, H.R. Sepangi and S. Shahidi, The scalar Einstein-aether theory, arXiv: 1404.7689 [INSPIRE].

[45] A.O. Barvinsky, Dark matter as a ghost free conformal extension of Einstein theory, JCAP 01 (2014) 014 [arXiv:1311.3111] [INSPIRE]. 
[46] R.L. Arnowitt, S. Deser and C.W. Misner, The Dynamics of general relativity, Gen. Rel. Grav. 40 (2008) 1997 [gr-qc/0405109] [INSPIRE].

[47] T.P. Sotiriou, M. Visser and S. Weinfurtner, Phenomenologically viable Lorentz-violating quantum gravity, Phys. Rev. Lett. 102 (2009) 251601 [arXiv:0904.4464] [INSPIRE].

[48] T.P. Sotiriou, M. Visser and S. Weinfurtner, Quantum gravity without Lorentz invariance, JHEP 10 (2009) 033 [arXiv:0905.2798] [INSPIRE].

[49] I. Kimpton and A. Padilla, Lessons from the decoupling limit of Hořava gravity, JHEP 07 (2010) 014 [arXiv: 1003.5666] [INSPIRE].

[50] A. Golovnev, On the recently proposed Mimetic Dark Matter, Phys. Lett. B 728 (2014) 39 [arXiv:1310.2790] [INSPIRE].

[51] K. Hammer and A. Vikman, Many Faces of Mimetic Gravity, arXiv:1512.09118 [INSPIRE].

[52] F. Arroja, N. Bartolo, P. Karmakar and S. Matarrese, The two faces of mimetic Horndeski gravity: disformal transformations and Lagrange multiplier, JCAP 09 (2015) 051 [arXiv: 1506.08575] [INSPIRE].

[53] F. Arroja, N. Bartolo, P. Karmakar and S. Matarrese, Cosmological perturbations in mimetic Horndeski gravity, JCAP 04 (2016) 042 [arXiv: 1512.09374] [INSPIRE].

[54] E.A. Lim, I. Sawicki and A. Vikman, Dust of Dark Energy, JCAP 05 (2010) 012 [arXiv: 1003.5751] [INSPIRE].

[55] L. Blanchet and S. Marsat, Modified gravity approach based on a preferred time foliation, Phys. Rev. D 84 (2011) 044056 [arXiv: 1107.5264] [InSPIRE].

[56] M. Bonetti and E. Barausse, Post-Newtonian constraints on Lorentz-violating gravity theories with a MOND phenomenology, Phys. Rev. D 91 (2015) 084053 [Erratum ibid. D 93 (2016) 029901] [arXiv: 1502.05554] [INSPIRE].

[57] T.-j. Chen, M. Fasiello, E.A. Lim and A.J. Tolley, Higher derivative theories with constraints: Exorcising Ostrogradski's Ghost, JCAP 02 (2013) 042 [arXiv: 1209.0583] [INSPIRE].

[58] R.P. Woodard, Avoiding dark energy with 1/r modifications of gravity, Lect. Notes Phys. 720 (2007) 403 [astro-ph/0601672] [INSPIRE].

[59] R.P. Woodard, Ostrogradsky's theorem on Hamiltonian instability, Scholarpedia 10 (2015) 32243 [arXiv: 1506.02210] [INSPIRE].

[60] V.F. Mukhanov, H.A. Feldman and R.H. Brandenberger, Theory of cosmological perturbations. Part 1. Classical perturbations. Part 2. Quantum theory of perturbations. Part 3. Extensions, Phys. Rept. 215 (1992) 203 [INSPIRE].

[61] D.S. Gorbunov and V.A. Rubakov, Introduction to the theory of the early universe: Cosmological perturbations and inflationary theory, World Scientific, Hackensack U.S.A. (2011) [INSPIRE].

[62] L.D. Landau and E.M. Lifshitz, The Classical Theory of Fields, Pergamon Press (1975).

[63] J.M. Pons, Substituting fields within the action: Consistency issues and some applications, J. Math. Phys. 51 (2010) 122903 [arXiv:0909.4151] [INSPIRE].

[64] M. Colombo, A.E. Gumrukcuoglu and T.P. Sotiriou, Hořava gravity with mixed derivative terms, Phys. Rev. D 91 (2015) 044021 [arXiv:1410.6360] [INSPIRE]. 
[65] M. Colombo, A.E. Gümrükçüoğlu and T.P. Sotiriou, Hořava gravity with mixed derivative terms: Power counting renormalizability with lower order dispersions, Phys. Rev. D 92 (2015) 064037 [arXiv: 1503.07544] [INSPIRE].

[66] A. Coates, M. Colombo, A.E. Gumrukcuoglu and T.P. Sotiriou, The uninvited guest in mixed derivative Hor̆ava Gravity, arXiv:1604.04215 [INSPIRE].

[67] M. Pospelov and Y. Shang, On Lorentz violation in Hořava-Lifshitz type theories, Phys. Rev. D 85 (2012) 105001 [arXiv: 1010.5249] [INSPIRE].

[68] B. Audren, D. Blas, M.M. Ivanov, J. Lesgourgues and S. Sibiryakov, Cosmological constraints on deviations from Lorentz invariance in gravity and dark matter, JCAP 03 (2015) 016 [arXiv:1410.6514] [INSPIRE].

[69] D. Blas, M.M. Ivanov and S. Sibiryakov, Testing Lorentz invariance of dark matter, JCAP 10 (2012) 057 [arXiv: 1209.0464] [INSPIRE].

[70] T. Jacobson, S. Liberati and D. Mattingly, Lorentz violation at high energy: Concepts, phenomena and astrophysical constraints, Annals Phys. 321 (2006) 150 [astro-ph/0505267] [INSPIRE].

[71] V.A. Kostelecky and N. Russell, Data Tables for Lorentz and CPT Violation, Rev. Mod. Phys. 83 (2011) 11 [arXiv:0801.0287] [inSPIRE].

[72] D. Blas and E. Lim, Phenomenology of theories of gravity without Lorentz invariance: the preferred frame case, Int. J. Mod. Phys. D 23 (2015) 1443009 [arXiv:1412.4828] [InSPIRE].

[73] S. Liberati, L. Maccione and T.P. Sotiriou, Scale hierarchy in Hor̆ava-Lifshitz gravity: a strong constraint from synchrotron radiation in the Crab nebula, Phys. Rev. Lett. 109 (2012) 151602 [arXiv: 1207.0670$]$ [INSPIRE].

[74] F. Sbisà, Classical and quantum ghosts, Eur. J. Phys. 36 (2015) 015009 [arXiv:1406.4550] [INSPIRE].

[75] R.C. Hickox and M. Markevitch, Absolute measurement of the unresolved cosmic X-ray background in the 0.5-8keV band with Chandra, Astrophys. J. 645 (2006) 95 [astro-ph/0512542] [INSPIRE].

[76] M. Turler, M. Chernyakova, T.J.L. Courvoisier, P. Lubinski, A. Neronov, N. Produit et al., INTEGRAL hard X-ray spectra of the cosmic X-ray background and Galactic ridge emission, Astron. Astrophys. 512 (2010) A49 [arXiv:1001.2110] [INSPIRE].

[77] EGRET collaboration, P. Sreekumar et al., EGRET observations of the extragalactic gamma-ray emission, Astrophys. J. 494 (1998) 523 [astro-ph/9709257] [INSPIRE].

[78] S.M. Carroll, M. Hoffman and M. Trodden, Can the dark energy equation - of - state parameter $w$ be less than -1?, Phys. Rev. D 68 (2003) 023509 [astro-ph/0301273] [INSPIRE].

[79] A.A. Klypin, A.V. Kravtsov, O. Valenzuela and F. Prada, Where are the missing Galactic satellites?, Astrophys. J. 522 (1999) 82 [astro-ph/9901240] [INSPIRE].

[80] B. Moore, S. Ghigna, F. Governato, G. Lake, T.R. Quinn, J. Stadel et al., Dark matter substructure within galactic halos, Astrophys. J. 524 (1999) L19 [astro-ph/9907411] [INSPIRE].

[81] T. Sawala et al., Local Group galaxies emerge from the dark, arXiv:1412.2748 [INSPIRE].

[82] G.N. Felder, L. Kofman and A. Starobinsky, Caustics in tachyon matter and other Born-Infeld scalars, JHEP 09 (2002) 026 [hep-th/0208019] [INSPIRE]. 
[83] E. Babichev, Formation of caustics in k-essence and Horndeski theory, JHEP 04 (2016) 129 [arXiv: 1602.00735] [INSPIRE].

[84] S. Ramazanov, Initial Conditions for Imperfect Dark Matter, JCAP 12 (2015) 007 [arXiv: 1507.00291] [INSPIRE].

[85] J. Chluba and R.A. Sunyaev, The evolution of CMB spectral distortions in the early Universe, Mon. Not. Roy. Astron. Soc. 419 (2012) 1294 [arXiv:1109.6552] [INSPIRE]. 